\title{
Using In situ Dynamic Cultures to Rapidly Biofabricate Fabric-Reinforced Composites of Chitosan/Bacterial Nanocellulose for Antibacterial Wound Dressings
}

\author{
Peng Zhang ${ }^{1}$, Lin Chen ${ }^{1}$, Qingsong Zhang ${ }^{1}$ and Feng F. Hong ${ }^{1,2 *}$ \\ ${ }^{1}$ Group of Microbiological Engineering and Industrial Biotechnology, College of Chemistry, Chemical Engineering and \\ Biotechnology, Donghua University, Shanghai, China, ${ }^{2}$ Key Laboratory of High Performance Fibers and Products, Ministry of \\ Education, Donghua University, Shanghai, China
}

OPEN ACCESS

Edited by:

Cheng Zhong,

Tianjin University of Science and

Technology, China

Reviewed by:

Amy Michele Grunden,

North Carolina State University, USA

Engy Ahmed,

Stockholm University, Sweden

${ }^{*}$ Correspondence:

Feng F. Hong

fhong@dhu.edu.cn

Specialty section:

This article was submitted to

Microbiological Chemistry and

Geomicrobiology,

a section of the journal

Frontiers in Microbiology

Received: 13 November 2015

Accepted: 16 February 2016

Published: 04 March 2016

Citation:

Zhang P, Chen L, Zhang Q and Hong FF (2016) Using In situ Dynamic

Cultures to Rapidly Biofabricate

Fabric-Reinforced Composites of

Chitosan/Bacterial Nanocellulose for Antibacterial Wound Dressings.

Front. Microbiol. 7:260.

doi: 10.3389/fmicb.2016.00260
Bacterial nano-cellulose (BNC) is considered to possess incredible potential in biomedical applications due to its innate unrivaled nano-fibrillar structure and versatile properties. However, its use is largely restricted by inefficient production and by insufficient strength when it is in a highly swollen state. In this study, a fabric skeleton reinforced chitosan (CS)/BNC hydrogel with high mechanical reliability and antibacterial activity was fabricated by using an efficient dynamic culture that could reserve the nano-fibrillar structure. By adding CS in culture media to $0.25-0.75 \%(\mathrm{w} / \mathrm{v})$ during bacterial cultivation, the CS/BNC composite hydrogel was biosynthesized in situ on a rotating drum composed of fabrics. With the proposed method, BNC biosynthesis became less sensitive to the adverse antibacterial effects of CS and the production time of the composite hydrogel with desirable thickness could be halved from 10 to 5 days as compared to the conventional static cultures. Although, its concentration was low in the medium, CS accounted for more than $38 \%$ of the CS/BNC dry weight. FE-SEM observation confirmed conservation of the nano-fibrillar networks and covering of CS on BNC. ATR-FTIR showed a decrease in the degree of intra-molecular hydrogen bonding and water absorption capacity was improved after compositing with CS. The fabric-reinforced CS/BNC composite exhibited bacteriostatic properties against Escherichia coli and Staphylococcus aureus and significantly improved mechanical properties as compared to the BNC sheets from static culture. In summary, the fabric-reinforced CS/BNC composite constitutes a desired candidate for advanced wound dressings. From another perspective, coating of BNC or CS/BNC could upgrade the conventional wound dressings made of cotton gauze to reduce pain during wound healing, especially for burn patients.

Keywords: bacterial cellulose, chitosan, composite sheet, antibacterial wound dressing, horizontal rotating bioreactor, in situ dynamic culture technology 


\section{INTRODUCTION}

Bacterial nano-cellulose (BNC) is a natural cellulosic material, which is mainly secreted by acetic acid bacteria. The appearance of BNC heralds a green approach to obtain cellulose from the microbiological industry, a useful supplement to the agroforestry industry (Klemm et al., 2005). BNC has more value other than a simple raw material because it is not just used as a new alternative cellulosic source, but also is an unparalleled functional material for its decent biocompatibility, nano-fibrillar supramolecular structure, hydrogel property, and super-high specific surface area, which is distinct from plant cellulose (Gatenholm and Klemm, 2010). Among them, the nano-fibrillar reticulate structure of microbially manufactured BNC is beyond what can currently be achieved artificially, and it looks like natural collagen in terms of its nanostructure and morphology, which is attractive for cell immobilization and because it mimics extracellular matrix (ECM) support (Petersen and Gatenholm, 2011). The current popular approach for fabrication of BNC composites destroys the innate nano-fibrous microstructure of BNC, which would impair its functionality that is based on its native morphology. Thus, conservation of BNC's natural nano $3 \mathrm{D}$ network structure and its corresponding features is an important consideration in the development of different functional BNC composites. Owing to BNC's unique bacterial cultivation procedure, a methodology of in situ static biosynthesis is commonly applied to produce BNC composites (Phisalaphong and Jatupaiboon, 2008; Wesarg et al., 2012). Specifically, in the in situ bio-synthesis process, the composites are generated through adding various materials into culture media during or before cultivation instead of using any postprocessing to alter an existing BNC hydrogel, for instance, to make other substances integrated into the BNC hydrogel matrix. As previously reported, the added materials may include soluble polymers (Phisalaphong and Jatupaiboon, 2008), particles (Yan et al., 2008), fibers (Pommet et al., 2008), and even some block fabrics (Meftahi et al., 2010).

Recently, BNC has been reported to possess a natural and intrinsic power to heal wounds (Czaja et al., 2006). Diverse BNC-based wound dressings, skin substitutes, and skin tissue repair materials have been developed to treat different traumas, such as burns (Fontana et al., 1990; Czaja et al., 2007), chronic wounds (Serafica et al., 2010), and full thickness skin injury (Fu et al., 2012). BNC is also favored in the development of various implanted medical devices, which include artificial menisci (Bäckdahl et al., 2006), vascular grafts (Bäckdahl et al., 2011; Hong et al., 2015; Tang et al., 2015), articular cartilages (Lopes et al., 2011; Martínez Ávila et al., 2014), and scaffolds for tissue regeneration (Bodin et al., 2010; Zaborowska et al., 2010). To satisfy miscellaneous applications, BNC has to be modified to improve its mechanical properties (Nakayama et al., 2004; Brown et al., 2011), rehydration abilities (Lin et al., 2009; Chen et al., 2011), antibacterial activity (Phisalaphong and Jatupaiboon, 2008), biocompatibility (Ciechańska, 2004; Cai and Kim, 2010), and diverse biological functions (Phisalaphong and Jatupaiboon, 2008). Among the properties, the mechanical strength to endure stress in actual use and the antibacterial activity to reduce infection risks are two general demands for most biomedical applications.

Although, the high number of intramolecular and intermolecular hydrogen bonds make the tensile strength of BNC much higher than many other natural materials, BNC itself is still unable to completely meet the requirements of wet wound dressings (Clasen et al., 2006) or the demands of other applications that need excellent mechanical performance (Nakayama et al., 2004). The mechanical properties of BNC hydrogel can be strengthened through reducing the porosity or lowing water content (Clasen et al., 2006; Retegi et al., 2010), compositing with other polymers to form a double network (Nakayama et al., 2004; Hagiwara et al., 2010), or cross-linking via glyoxalization (Quero et al., 2011), all of which certainly alter the inherent structure or chemical composition of BNC hydrogel. Reinforcing hydrogel with a fabric skeleton, by contrast, is a desirable approach to significantly enhance the mechanical properties without dramatically altering the native features of hydrogels (Agrawal et al., 2013). For instance, involvement of fabrics remarkably improved tear resistance of poly(2-hydroxyethyl methacrylate; pHEMA) hydrogel for artificial skin use (Young et al., 1998). Since, conventional hydrogels are formed from monomer solutions or dispersive polymer suspensions, it is easy to embed a frame network into gel matrix through immersing fabrics in raw polymer solutions or monomer solutions followed by cross-linking or polymerization. An analogous approach used for embedding fabric skeletons into BNC hydrogel is to place the fabrics in culture media directly. However, under current static culture conditions, the fabrics as skeletons must float on the surface of culture media since BNC is only excreted to near the air-liquid interface $(<1 \mathrm{~mm}$ in depth; Verschuren et al., 2000; Hornung et al., 2006), which leads to the fabric being half-encapsulated and half-unmodified in one culture cycle (Meftahi et al., 2010).

Chitosan (CS), a natural amino polysaccharide, possesses many outstanding properties, such as the biodegradability, biocompatibility, non-toxicity, healing promotion, and especially antibacterial properties. Therefore, it is highly promising to combine it with BNC to develop ideal functional composites for biomedical applications. The CS/BNC composites have been fabricated in various ways, such as impregnating the purified BNC matrix in CS solution (Kim et al., 2011; UlIslam et al., 2011; Lin et al., 2013), casting of CS-blended BNC homogenate suspension (Fernandes et al., 2009; Nge et al., 2010) or casting of CS-blended BNC co-solution (Wu et al., 2004), and in situ static bio-synthesis (Ciechańska, 2004; Phisalaphong and Jatupaiboon, 2008). Most results showed CS/BNC composites gained antibacterial effects and improved other properties including moisture retention, and bioactivity. By using the impregnation method, CS penetrates completely into BNC matrix via free and physical diffusion, which needs a prolonged period for soaking and depends on molecular weight and concentration of CS, as well as pore sizes of BNC network. The padded CS in the original interstices of BNC would possibly be lost by subsequent diffusion in practical applications. In the casting approach, the inherent microstructures of BNC hydrogel will be entirely disrupted. Therefore, the in situ biosynthesis 
method, where chitosan is supplied in bacterial cultures, is supposed to be better to develop CS/BNC composites. However, the antimicrobial property of chitosan may inhibit the bacterial growth and decrease cellulose production, resulting in very thin CS/BNC sheets (Ciechańska, 2004). Besides, the crude BNC composites obtained by in situ biosynthesis have to be subjected to a routine purification process composed of a series of alkaline boiling and water washing steps to achieve a medical grade nonpyrogenic material before clinical uses (McKenna et al., 2009; Martínez Ávila et al., 2014). During the purification process, the loosely bound CS could be completely removed from the BNC matrix (Heßler and Klemm, 2009).

In order to solve the problems with preparation of the CS/BNC composites in static cultures, dynamic cultures were therefore proposed to produce fabric-embedded CS/BNC hydrogel sheets in a horizontal rotating bioreactor. In this approach, cotton gauze used as a mode fabric was fixed to form a rotating drum to immobilize $\mathrm{CS} / \mathrm{BNC}$ hydrogel as the support during bacterial cultivation. By compositing, the embedded fabric functioned as the inner skeleton of the composite to reinforce the hydrogel. This process was expected to minimize the negative antibacterial impacts of chitosan during cultivation. In situ dynamic biosynthesis of CS/BNC composite sheets was performed in the presence of three CS addition concentrations $(0.25,0.50$, and $0.75 \%, w / v)$ and was compared with the conventional $\mathrm{CS} / \mathrm{BNC}$ composites from static cultures. The characteristics including tensile properties, water holding and absorption properties, and antibacterial properties of the fabricreinforced $\mathrm{CS} / \mathrm{BNC}$ composites were investigated in detail.

\section{MATERIALS AND METHODS}

\section{Microorganism}

The bacterial strain used in this study was Gluconacetobacter xylinus ATCC 23770 (obtained from American Type Culture Collection, Manassas, VA), which was preserved on agar slant at $4^{\circ} \mathrm{C}$. One loop of colonies was inoculated into a culture medium containing 2.5\% (w/v) D-mannitol, $0.5 \%(\mathrm{w} / \mathrm{v})$ yeast extracts, $0.3 \%(\mathrm{w} / \mathrm{v})$ peptone with an acetic acid-adjusted $\mathrm{pH}$ of 5.0 , and then cultivated with a shaking speed of $160 \mathrm{rpm}$ at $30^{\circ} \mathrm{C}$ for $12 \mathrm{~h}$ to prepare seed culture.

\section{Preparation of Fabric-Reinforced CS/BNC Composites \\ Preparation of Culture Medium}

The chitosan with viscosity of 50-800 $\mathrm{mPa} \cdot \mathrm{s}$ (average $\mathrm{MW}=620 \mathrm{kDa}$ ) and degree of deacetylation 92\% (Sinopharm Chemical Reagent Co., Ltd, Shanghai, China) was dissolved in diluted acetic acid to reach a concentration $(\mathrm{w} / \mathrm{v})$ of $0.25,0.5$, and $0.75 \%$ separately, and then was premixed with $2.5 \%(\mathrm{w} / \mathrm{v})$ glucose, $0.5 \%(\mathrm{w} / \mathrm{v})$ yeast extract, $0.3 \%(\mathrm{w} / \mathrm{v})$ peptone. Higher concentration of chitosan-added medium was not achieved because of co-dissolving difficulties with the culture substrates. The chitosan-free medium with the same formula was used as the control. The $\mathrm{pH}$ value in all the culture media was adjusted to 4.5 with acetic acid before autoclaved at $121^{\circ} \mathrm{C}$ for $20 \mathrm{~min}$.

\section{Design of the Horizontal Rotating Bioreactor}

A laboratory-scale horizontal rotating bioreactor was constructed and applied in this study. As shown in Figure 1, the bioreactor is equipped with a horizontal glass cylinder as its container and a rotating roller cage $(9.0 \mathrm{~cm}$ in diameter) used to fasten fabric (Hong et al., 2012a). The roller cage is rotated via a central spindle connected to a controllable motor. The top half of glass cylinder could be opened to remove or replace the roller cage. The dry weight of cotton gauzes was pre-weighed before installation onto roller cage for cultivation. The fabric-equipped bioreactor was autoclaved without media at $121^{\circ} \mathrm{C}$ for $20 \mathrm{~min}$ and then placed in a sterile safety cabinet before inoculation.

\section{Preparation of CS/BNC Composites}

The fabric-reinforced CS/BNC composites in rotating cultures (Hong, 2012b) and the CS/BNC composite sheets in static cultures were prepared, respectively, following the procedures as shown in Figure 1. Rotating cultivation was performed by transferring $400 \mathrm{~mL}$ inoculated culture medium into the horizontal bioreactor with $10 \%(\mathrm{v} / \mathrm{v})$ inocula and then cultivated with an optimized rotating speed of $25 \mathrm{rpm}$ at $30^{\circ} \mathrm{C}$ for 5 days. Four parallel cultures were carried out for each CS concentration of $0,0.25,0.5$, and $0.75 \%$. After cultivation, the fabric-reinforced $\mathrm{CS} / \mathrm{BNC}$ composite sheets were harvested from the roller. At the same time, a static cultivation in quadruplicate was carried out in $250 \mathrm{~mL}$ flasks containing $100 \mathrm{~mL}$ inoculated media for 10 days as the control. The number of viable bacterial cells in the liquid media was monitored through serial dilutions and spread plating.

\section{Purification and Gravimetric Analysis of CS/BNC Composites}

A routine harsh purification procedure (McKenna et al., 2009) was employed in this research, including incubation in $0.5 \%$ $(\mathrm{w} / \mathrm{v}) \mathrm{NaOH}$ aqueous solution (renewed for each $2 \mathrm{~h}$ ) at $80^{\circ} \mathrm{C}$ for $8 \mathrm{~h}$ to remove bacterial cells and other ingredients, followed by rinsing with deionized water until $\mathrm{pH}$ was neutral.

The purified samples were completely dried at $105^{\circ} \mathrm{C}$ to a constant weight for gravimetrical measurements. The total dry weight of $\mathrm{BNC}$ or $\mathrm{CS} / \mathrm{BNC}$ coat of the composites from rotating cultivations was obtained by subtracting the dry weight of cotton gauze from the total weight of cotton gauze-reinforced $\mathrm{BNC}$ or $\mathrm{CS} / \mathrm{BNC}$ composites. The total dry mass of BNC or $\mathrm{CS} / \mathrm{BNC}$ hydrogels from static cultivations was directly obtained by gravimetrical measurements.

\section{Characterization of CS/BNC Composites Microstructure Inspection}

The reinforced CS/BNC and the pristine BNC were freeze-dried in swollen state and were sprayed with gold for observation. The microstructure of the samples was observed with a field emission scanning electron microscope (FE-SEM, S-4800 model, Hitachi, Japan).

\section{Thermogravimetric Analysis}

The CS/BNC coat layer was stripped with a lancet from a reinforced CS/BNC composite sheet after freeze-dried. The $\mathrm{CS} / \mathrm{BNC}$ coat sample of 3-5 mg was weighed and loaded into an 


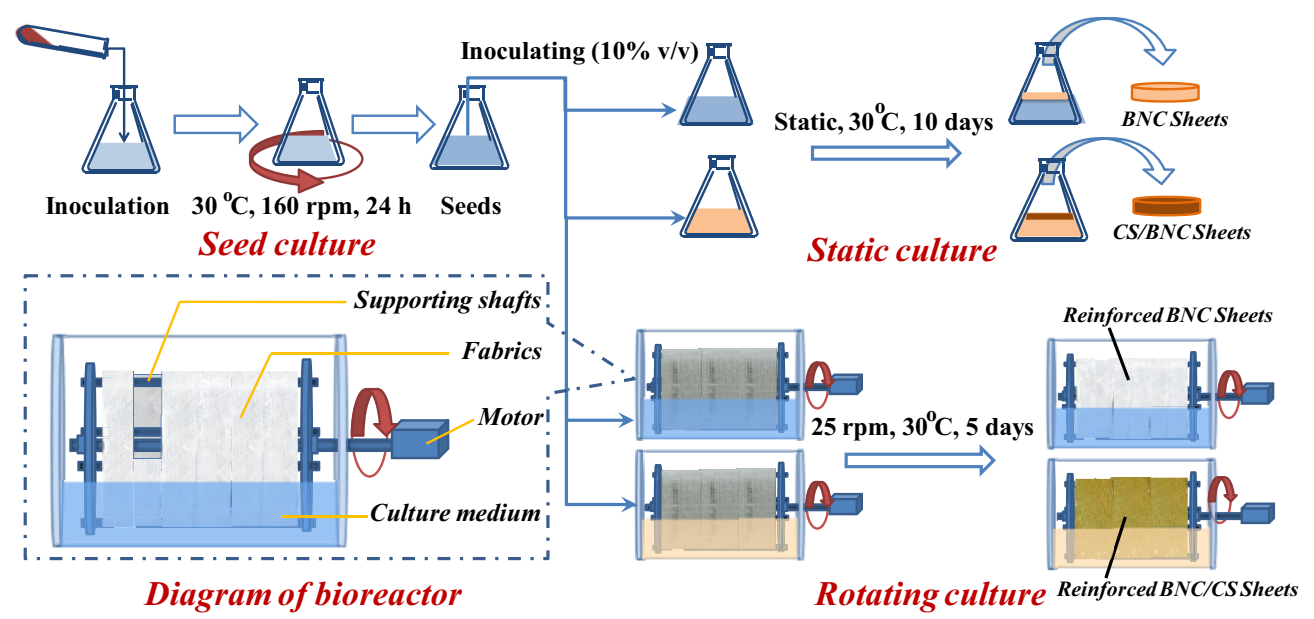

FIGURE 1 | Preparation processes of the CS/BNC composites with rotating culture and static culture.

alumina oxide pan to carry out the thermal gravimetric analysis (TGA) by using a Netzsch TG-209-F1 Libra gravimetric analyzer (Netzsch, Germany). The samples were heated from 25 to $900^{\circ} \mathrm{C}$ with a heating rate of $10 \mathrm{~K} \mathrm{~min}^{-1}$ under the protection of nitrogen flow $\left(250 \mathrm{~mL} \mathrm{~min}^{-1}\right)$.

\section{Content of Chitosan}

The content of chitosan in the CS/BNC composite coat (excluding fabric skeleton) was obtained using the elemental analysis method. Nitrogen percentage of CS/BNC composite $\left(\mathrm{N}_{\mathrm{CS} / \mathrm{BNC}}\right)$, of chitosan $\left(\mathrm{N}_{\mathrm{CS}}\right)$, and of $\mathrm{BNC}\left(\mathrm{N}_{\mathrm{BNC}}\right)$ was detected with an elemental analyzer (Vario EL III, Elementar Analyser Systems GmbH, Hanau, Germany). Both total mass and total nitrogen of CS/BNC composite are supposed to be assigned to $\mathrm{CS}$ portion and BNC portion:

$$
\begin{gathered}
\text { Total } \mathrm{m}_{\mathrm{CS} / \mathrm{BNC}}=\mathrm{m}_{\mathrm{CS}}+\mathrm{m}_{\mathrm{BNC}} \\
\text { Total } \mathrm{N}_{\mathrm{CS} / \mathrm{BNC}}=\mathrm{P}_{\mathrm{CS}}+\mathrm{P}_{\mathrm{BNC}}
\end{gathered}
$$

where, Total $\mathrm{m}_{\mathrm{CS} / \mathrm{BNC}}, \mathrm{m}_{\mathrm{CS}}$, and $\mathrm{m}_{\mathrm{BNC}}$ is the total mass of $\mathrm{CS} / \mathrm{BNC}$ composite, the mass of $\mathrm{CS}$ and BNC component, respectively. Total $\mathrm{N}_{\mathrm{CS} / \mathrm{BNC}}$ is the total nitrogen in CS/BNC composite that is composed of CS portion $\left(\mathrm{P}_{\mathrm{CS}}\right)$ and $\mathrm{BNC}$ portion ( $\mathrm{P}_{\mathrm{BNC}}$, although very little). Thus, the element conservation Equation (2) of nitrogen can be specified as:

$$
\begin{aligned}
& \text { Total } \mathrm{N}_{\mathrm{CS} / \mathrm{BNC}}=\mathrm{N}_{\mathrm{CS} / \mathrm{BNC}} \times \mathrm{m}_{\mathrm{CS} / \mathrm{BNC}}=\mathrm{P}_{\mathrm{CS}}+\mathrm{P}_{\mathrm{BNC}} \\
& =\mathrm{N}_{\mathrm{CS}} \times \mathrm{m}_{\mathrm{CS}}+\mathrm{N}_{\mathrm{BNC}} \times \mathrm{m}_{\mathrm{BNC}} \\
& =\mathrm{N}_{\mathrm{CS}} \times \mathrm{m}_{\mathrm{CS}}+\mathrm{N}_{\mathrm{BNC}} \times\left(\mathrm{m}_{\mathrm{CS} / \mathrm{BNC}}-\mathrm{m}_{\mathrm{CS}}\right)
\end{aligned}
$$

CS content $\left(\alpha_{c s}\right)$ in the CS/BNC composite was calculated with the following rearranged equation:

$$
\mathrm{CS} \text { content }\left(\alpha_{\mathrm{CS}}\right)=\frac{\mathrm{m}_{\mathrm{CS}}}{\mathrm{m}_{\mathrm{CS} / \mathrm{BNC}}}=\frac{\mathrm{N}_{\mathrm{CS} / \mathrm{BNC}}-\mathrm{N}_{\mathrm{BNC}}}{\mathrm{N}_{\mathrm{CS}}-\mathrm{N}_{\mathrm{BNC}}} \times 100 \%
$$

\section{ATR/FT-IR}

Molecular structure variations of CS/BNCs were investigated with an infrared spectrophotometer equipped with attenuated total reflectance (FTIR-8400, Shimadzu Co. Japan) and the spectra from 4500 to $600 \mathrm{~cm}^{-1}$ were recorded.

\section{Water Holding and Absorption Characteristics}

The water absorption capacity was measured according to the British Pharmacopeia monograph for alginate dressings and packing. The hydrogel samples were cut to rectangles of $2 \times$ $4 \mathrm{~cm}$ sizes and were weighed as initial weight $\left(\mathrm{W}_{0}\right)$, after that the samples were freeze-dried and then were soaked in solution A (containing $2.5 \mathrm{mmol} / \mathrm{L} \mathrm{CaCl}_{2} \cdot 2 \mathrm{H}_{2} \mathrm{O}$ and $142 \mathrm{mmol} / \mathrm{L}$ $\mathrm{NaCl}$ which simulate the ionic strength of $\mathrm{Ca}^{2+}$ and $\mathrm{Na}^{+}$in wound exudates) for $30 \mathrm{~min}$. The wet samples were weighed $\left(\mathrm{W}_{1}\right)$ after picked out and hung up with a forceps for $30 \mathrm{~s}$ to remove the surface liquid. After that, the samples were transferred to a centrifuge tube whose bottom was padded knitted gauze to absorb squeezed water. The samples were centrifugally dehydrated for $15 \mathrm{~min}$ at $1200 \mathrm{rpm}$ and were weighted $\left(\mathrm{W}_{2}\right)$. The ultimate weight of completely dried samples $\left(\mathrm{W}_{3}\right)$ was weighed after drying at $105^{\circ} \mathrm{C}$. The water holding capacity (WHC) and water absorption capacity (WAC) was calculated as:

$$
\begin{aligned}
& \mathrm{WHC}=\frac{\mathrm{W}_{0}-\mathrm{W}_{3}}{\mathrm{~W}_{3}} \\
& \mathrm{WAC}=\frac{\mathrm{W}_{1}-\mathrm{W}_{3}}{\mathrm{~W}_{3}}
\end{aligned}
$$

Fluid held within the sample was divided into two parts: fluid held between fibers $\left(\mathrm{W}_{1}-\mathrm{W}_{2}\right)$ and fluid held inside the individual fibers $\left(\mathrm{W}_{2}-\mathrm{W}_{3}\right)$. And the normalized parameters $\left(\mathrm{W}_{1}-\mathrm{W}_{2}\right) / \mathrm{W}_{3}$ and $\left(\mathrm{W}_{2}-\mathrm{W}_{3}\right) / \mathrm{W}_{3}$, which expressed the fluid intake of per gram sample $(\mathrm{g} / \mathrm{g})$, were used to compare dressing materials (Qin, 2004). The ratio of $\left(\mathrm{W}_{1}-\mathrm{W}_{2}\right) /\left(\mathrm{W}_{2}-\mathrm{W}_{3}\right)$ was also evaluated, indicating distribution of fluid in dressing (Qin, 2004, 2008). Each test was repeated eight times, and then mean values \pm standard deviations were given. 


\section{Antibacterial Performance Tests}

Considering both wet and dry BNC materials (i.e., hydrogel and dehydrated $\mathrm{BNC}$ ) have the respective advantages in therapy of chronic traumas and burns (Fu et al., 2013), and treatment of acute traumas (Wei et al., 2011), the antibacterial performances of the fabric-reinforced samples both in hydrogel state and lyophilized state against Escherichia coli and Staphylococcus aureus were evaluated. Two quantitative evaluation methods, the absorption method (Figure 2A) and the shake flask method (saline, Figure 2B) were applied. In the former method, the bacterial suspension immediately touched with the samples by liquid absorption, while in the shake flask method the composite samples were cultured in a dynamic germy liquid culture medium by shaking. A gauze-reinforced BNC sample was used as control. The test procedures are described as follows:

\section{(A). Absorption method (ISO 20743-2007)}

In the absorption method (Figure 2A), the bacterial inoculum directly contacted with control and test samples by pipetting inocula on the surface of samples. As shown in Figure 2A, the absorption method was performed according to ISO20743-2007 standard. An adjusted germy inoculum $\left(1 \times 10^{5}\right.$ to $\left.3 \times 10^{5} \mathrm{cfu} / \mathrm{mL}\right)$ of $0.2 \mathrm{~mL}$ was scattered on the sample surface in a vial and tighten the cap. After touching with the sample for $24 \mathrm{~h}, 20 \mathrm{~mL}$ Soya Casein Digest Lecithin Polysorbate (SCDLP) base broth medium, consisting of $17 \mathrm{~g} / \mathrm{L}$ peptone from casein, $3 \mathrm{~g} / \mathrm{L}$ soy peptone, 5 $\mathrm{g} / \mathrm{L} \mathrm{NaCl}, 2.5 \mathrm{~g} / \mathrm{L} \mathrm{KH}_{2} \mathrm{PO}_{3}, 2.5 \mathrm{~g} / \mathrm{L}$ glucose, $1 \mathrm{~g} / \mathrm{L}$ lecithin, and $7 \mathrm{~g} / \mathrm{L}$ polysorbate 80 , was added into the vial and washed out the cells in the samples using a vortex mixer. The number of viable cells was quantified by spread plating of serial dilutions. The gauze-reinforced BNC samples in hydrogel and lyophilized states were used as controls and each sample had three parallels. The percentage reduction $(\mathrm{R})$, growth value of control $(\mathrm{F})$, and antibacterial activity value of test (A) were calculated as follows:

$$
\begin{aligned}
\mathrm{R}(\%) & =\frac{\mathrm{C}_{\mathrm{t}}-\mathrm{T}_{\mathrm{t}}}{\mathrm{C}_{\mathrm{t}}} \times 100 \% \\
\mathrm{~F} & =\lg \mathrm{C}_{\mathrm{t}}-\lg \mathrm{C}_{0} \\
\mathrm{~A} & =\lg \mathrm{T}_{\mathrm{t}}-\lg \mathrm{C}_{\mathrm{t}}
\end{aligned}
$$

where, $C_{t}, T_{t}$, and $C_{0}$ was the average concentration of bacterial cells of control sample at $24 \mathrm{~h}$, of test sample at $24 \mathrm{~h}$, and of control sample at $0 \mathrm{~h}$, respectively.

(B). Shake-flask method (saline)

As shown in Figure 2B, the shake-flask method (saline) was performed according to Singh's method (Singh et al., 2012). The sample of $0.75 \mathrm{~g}$ was cut into small pieces, and then soaked in a flask containing $70 \mathrm{~mL}$ PBS and $5 \mathrm{~mL}$ germy medium $\left(3 \times 10^{5}\right.$ to $3 \times 10^{6} \mathrm{cfu} / \mathrm{mL}$ ). Then the flask was cultivated with a shaking speed of $180 \mathrm{rpm}$ at $37^{\circ} \mathrm{C}$ for E. coli and S. aureus. The number of viable cells in the shake-flask after inoculation $(0 \mathrm{~h})$ and shaking for $24 \mathrm{~h}$ was detected using spread plating of serial dilutions. The gauze-reinforced BNC samples in hydrogel and lyophilized states were used as controls and each test was performed in three parallels. The percentage reduction (R), growth value of control
(F), and antibacterial activity value of test (A) were calculated as the same as that in the absorption method.

\section{Mechanical Properties}

The water-saturated samples were cut into rectangles of $1 \times$ $4 \mathrm{~cm}$. The tensile evaluation was performed on a universal material testing machine (H5K-S, Hounsfield Test Equipment Ltd., England) under ambient condition $\left(25^{\circ} \mathrm{C}\right.$, around $50 \%$ relative humidity) at a speed of $50 \mathrm{~mm} / \mathrm{min}$ with a $100 \mathrm{~N}$ sensor loaded. Each test was repeated eight times, and then mean values \pm standard deviation were given.

\section{Statistic}

In the analysis of the mechanical and antibacterial properties, the statistical significance was tested by one-way analysis of variance (ANOVA). A post-hoc test (Tukey HSD) was applied to specify differences among means. $P<0.05$ was considered as significant.

\section{RESULTS AND DISCUSSION \\ Bio-Fabrication of Fabric-Reinforced BNC and CS/BNC Composites}

$\mathrm{CS} / \mathrm{BNC}$ composite sheets were bio-fabricated in situ by using both static culture and rotating culture (as shown in Figure 1). Chitosan solution is a usually transparent and pale yellow liquid but turns brown after autoclaving sterilization due to Maillard reactions between $-\mathrm{NH}_{2}$ and $-\mathrm{OH}$ groups (Yang et al., 2007), which could bring about an increasing color intensity in both the culture broth and the resulting hydrogel sheets (as shown in Figures $3 \mathbf{a}, \mathbf{b})$. In the static culture, the composite sheets floated on the surface of culture broth, while in the rotating culture the fabric-reinforced composite sheets formed via rotation coating and in situ biosynthesis of BNC on the fabric skeleton. In the former situation, the production efficiency of pristine BNC was far lower than that in the rotating culture, which should be ascribed to defects in mass transfer (Hornung et al., 2006). Therefore, a longer cultivation period of $10 \mathrm{~d}$ was required for static culture to obtain the thickness of pristine BNC sheet of $2.50 \pm 0.35 \mathrm{~mm}$, while only $5 \mathrm{~d}$ rotating cultivation was needed for the fabric-reinforced BNC sheet to achieve similar thickness $(2.49 \pm 0.32 \mathrm{~mm})$. With addition of chitosan to culture medium to low concentration $(0.25-0.75 \%$, w/v), the production of hydrogel sheets in the static culture was strongly inhibited and sheet thickness decreased drastically (Figure 3a). $\mathrm{CS} / \mathrm{BNC}$ composite sheets produced in static culture had a regular appearance on the air interface side, but the underside of the sheets adhered only as a loose gel that was weak and formless. This gel could be completely removed in a subsequent alkaline purification treatment. The thicknesses of iCS/BNC ( $i$ was $0.25,0.50,0.75 \%$, respectively) sheets obtained from the static cultures were all less than $0.10 \mathrm{~mm}$ after purification, and their volume dry weights were $0.24,0.35,0.51 \mathrm{~g} / \mathrm{L}$ for $0.25 \% \mathrm{CS} / \mathrm{BNC}, 0.50 \% \mathrm{CS} / \mathrm{BNC}$, and $0.75 \% \mathrm{CS} / \mathrm{BNC}$, respectively. By element analysis (EA), the detected nitrogen percentage in the pristine $\mathrm{BNC}$ was lower than $0.12 \%(\mathrm{w} / \mathrm{w})$, indicating residues of cells and culture medium in the BNC matrix were little 


\section{A Absorption method}

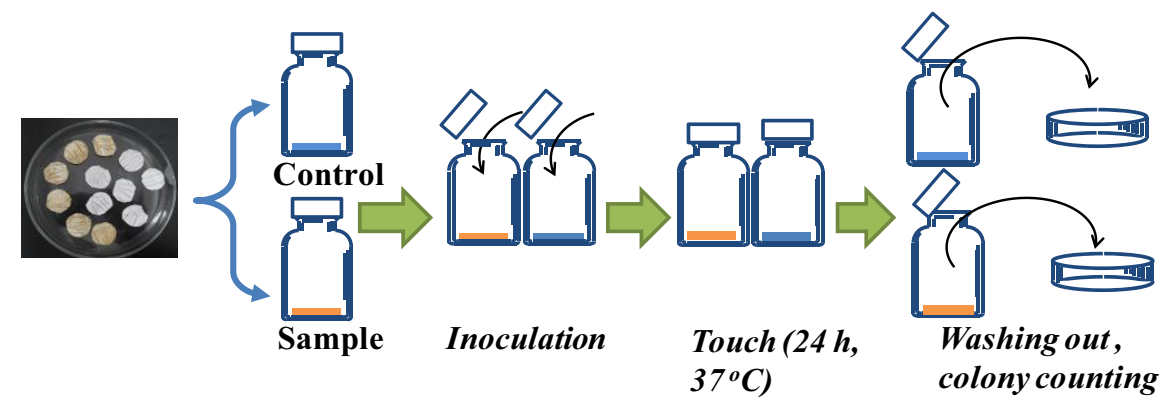

B Shake-flask method

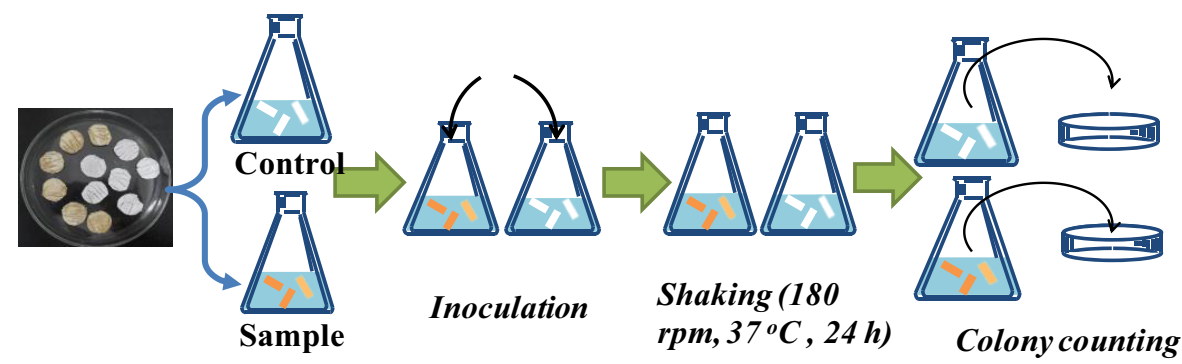

FIGURE 2 | Protocol of the antibacterial ability test: (A) absorption method (ISO 20743-2007), (B) shake-flask method (saline).

after the alkaline purification. Thus, the deductive CS content in the dry CS/BNC composite coat (excluding fabric) based on nitrogen percentage was determined as $48.24,39.96$, and $35.54 \%$ $(\mathrm{w} / \mathrm{w})$ for $0.25 \% \mathrm{CS} / \mathrm{BNC}, 0.50 \% \mathrm{CS} / \mathrm{BNC}$, and $0.75 \% \mathrm{CS} / \mathrm{BNC}$, respectively, which was $0.12,0.22$, and $0.33 \mathrm{~g} / \mathrm{L}$ corresponding to the absolute yield of composite (Figure $3 \mathbf{b}$ ). Surprisingly, the absolute yield of composite rose slightly with the increase of chitosan addition from 0.25 to $0.75 \%(\mathrm{w} / \mathrm{v})$. One possible reason for the increase could be the addition of acetic acid that was used for dissolving chitosan, which could be utilized as a carbon source for G. xylinus. Previous studies have shown that acetic acid can enhance BNC yield (Yang et al., 2014). However, the cellulose production was much lower after adding chitosan than that in the chitosan-free culture medium $(1.46 \mathrm{~g} / \mathrm{L})$, indicating a serious inhibiting effect on cellulose production under static culture condition. Figure $3 \mathrm{c}$ also shows that growth of G. xylinus was inhibited since the cell density decreased in the initial $24 \mathrm{~h}$ after inoculation, but could rebound in the next $48 \mathrm{~h}$, indicating an adaptiveness of G. xylinus against low concentration of CS. Since the CS/BNC composite sheets obtained from static cultures were too fragile and thin to accomplish thorough evaluations (like mechanical tests or antibacterial tests), it is less possible for practical use and therefore was not evaluated further in this study.

By utilizing fabrics as the support in a self-designed horizontal rotating bioreactor (Hong et al., 2012a; Zhang et al., 2013), $\mathrm{BNC}$ or CS/BNC hydrogel sheets were satisfactorily developed through in situ biosynthesis on the fabrics. The appearance of fabric-reinforced CS/BNC and -reinforced BNC composites after purification was shown in Figure 3A. Unlike the CS/BNC sheets obtained in static cultures, dynamically-cultured CS/BNC hydrogel sheets showed compact, stable, and symmetrical appearances. The CS/BNC sheets had a fairly good thickness of $2.68 \pm 0.42,2.69 \pm 0.33$, and $2.84 \pm 0.45 \mathrm{~mm}$ for $0.25 \% \mathrm{CS} / \mathrm{BNC}$, $0.50 \% \mathrm{CS} / \mathrm{BNC}$, and $0.75 \% \mathrm{CS} / \mathrm{BNC}$, respectively, which was slightly higher than the reinforced pristine BNC sheets (2.49 $\pm 0.32 \mathrm{~mm})$. The fabric skeletons could be seen through the transparent hydrogel coat and the color of the iCS/BNC hydrogel coat changed from completely colorless transparency of pristine BNC to tawny with the increase of CS addition from 0.25 to $0.75 \%$. The coating amount of hydrogel was $30.75,56.41,61.81$, and $66.91 \mathrm{~g} / \mathrm{m}^{2}$, corresponding to the absolute output of 1.48 , $2.71,2.97$, and $3.21 \mathrm{~g} / \mathrm{L}$ (without the weight of fabrics), for the additive concentrations of chitosan of $0,0.25,0.50$, and $0.75 \%$, respectively. The CS content in the CS/BNC composite coat obtained with the CS addition of $0.25,0.50$, and $0.75 \%(\mathrm{w} / \mathrm{v})$ was $39.52,41.53$, and $43.27 \%$, respectively. The only cellulose output in the $0.25-0.75 \%$ CS-added medium was $1.63,1.73$, and $1.82 \mathrm{~g} / \mathrm{L}$, respectively, which increased with CS addition and was slightly higher than the CS-free medium $(1.48 \mathrm{~g} / \mathrm{L})$, as shown in Figure 3B. The results indicate that CS could be incorporated into the BNC successfully when it was produced in the rotating culture system, and the CS could be retained in the BNC matrix stably even after undergoing a harsh alkaline purification. Compared with the in situ static culture, the inhibiting effects on cellulose production were eliminated in the rotating culture. 


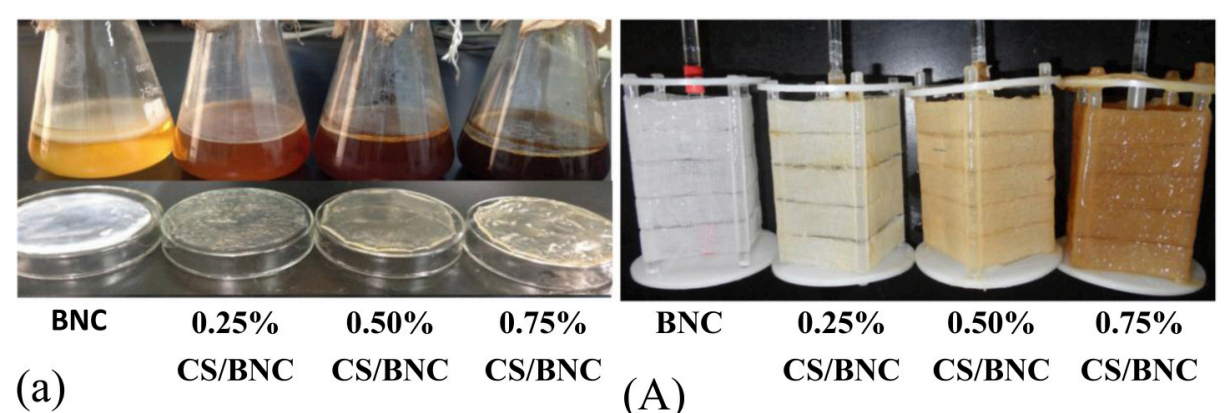

(a)
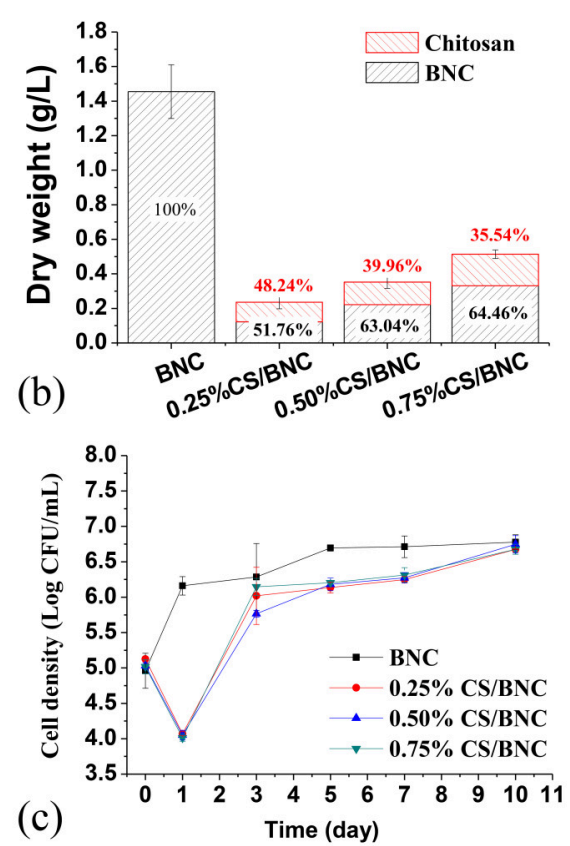

(A)
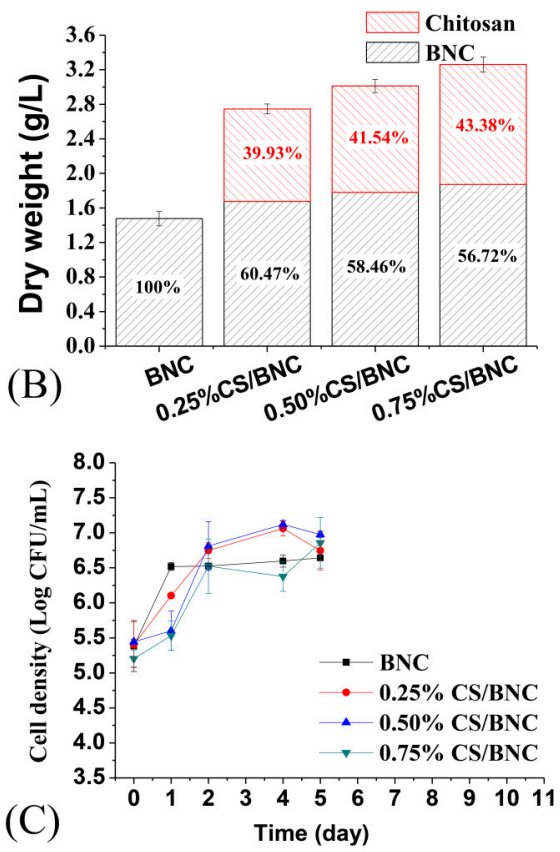

FIGURE 3 | Biosynthesis of the CS/BNC composites: appearance (after purification) of BNC and CS/BNC composites in static cultures (a) and fabric-reinforced BNC and CS/BNC composites in rotating cultures (A). The dried weight of BNC, CS/BNC sheets and composite components in static cultures and rotating cultures are show in (b,B), respectively. The cell growth curves in static cultures (c) and rotating cultures (C) are shown as well. The experiments were performed in triplicate, and standard deviations were given as error bars.

The cell growth curves in the rotating culture (Figure 3C) did not show the remarkable reduction that was found within $1 \mathrm{~d}$ in the static culture, although the growth rate in the CS-added culture was slower than the CS-free culture in the initial $24 \mathrm{~h}$ after inoculation. This result indicates that the present CS addition gave a low level inhibition effect on bacterial growth in the rotating culture.

CS is a natural antibacterial polymer, which can disrupt cytomembrane structure, cellular energy metabolism, and protein synthesis (Raafat et al., 2008; Galván Márquez et al., 2013). As CS is a dose-dependent bactericide (Raafat et al., 2008), addition of a small amount of CS into culture medium would not completely inhibit the cell proliferation and cellulose production, even could make CS incorporate into BNC matrix successfully during in situ biosynthesis. Static culture appeared to be more susceptible than rotating culture to the addition of CS. Under the negative influence of CS, only very thin BNC composite sheets (less than a quarter of BNC mass in the CS-free culture, Figure $3 \mathbf{b}$ ) could be harvested in the static cultures as reported in literature (Ciechańska, 2004), while the same effects were not observed in the rotating cultures (Figure 3B). One possible explanation is that rotating cultures provide a better nutrient and oxygen transport than static cultures, which is conducive to bacterial proliferation and metabolism (Serafica et al., 2002) and consequently enhances bacterial adverse-resistant activity. The other explanation is that the immobilization of BNC hydrogel on the fabric support is a natural form of cell immobilization, just like biofilm, which helps G. xylinus cells to achieve higher regional cell numbers and to resist the harmful circumstances (Cheng et al., 2009; Maksimova, 2014). Thus, compared with the static cultures, the bacteria were able to grow faster in the rotating cultures to achieve a higher cell density and to get a quick adaption to the CS-added cultures, which can be concluded from the growth curves (Figures 3c,C). 


\section{Characterization of Fabric-Reinforced BNC and CS/BNC Composites}

\section{Morphology}

Since it is difficult to prepare the fracture surface of the whole fabric-reinforced BNC composites for SEM inspection, the cross section of the hydrogel coat layer striped from the fabricreinforced $\mathrm{BNC}$ and $\mathrm{CS} / \mathrm{BNC}$ composites was examined with $\mathrm{FE}-$ SEM, as shown in Figure 4. Figures $4 a, A$ show a typical nanofibrous network of BNC, whose fiber diameters mostly range in a narrow scope between 20 and $60 \mathrm{~nm}$ with an average value of $39.1 \mathrm{~nm}$. With addition of $0.25,0.50$, and $0.75 \%(\mathrm{w} / \mathrm{v}) \mathrm{CS}$, the average size of single fiber increased to $54.9,41.8$, and $58.8 \mathrm{~nm}$, respectively. And the distribution of fiber diameters tends to turn much wider (Figures 4B-D). Distinct morphological difference between $\mathrm{BNC}$ and $\mathrm{CS} / \mathrm{BNC}$ was that the BNC fiber was fairly uniform, while CS/BNC fiber size was irregular even for single fiber. This may result from CS disturbing the normal aggregation of BNC fibrils as it incorporates into the BNC fibers. Besides distribution of fiber diameter, CS/BNC network was much denser than BNC. In contrast, CS/BNC composites prepared by soaking $\mathrm{BNC}$ sheets in a CS solution have massive visible depositions of CS that fill in empty spaces of fibrillar network of BNC or coat on the surface of BNC fibers (Kim et al., 2011; Ul-Islam et al., 2011), which dramatically alters the original morphology to a greater degree than the CS/BNC composites from the in situ rotating cultures. The FE-SEM results showed that CS was perfectly dispersed and composited with internal nano-fibers of BNC. Overall, hydrogel coat of the CS/BNC composites obtained in the rotating cultures remained a similar nano-fibrillar structure as pristine $\mathrm{BNC}$, and more importantly, multiple unique structurerelated features of $\mathrm{BNC}$ could also be remained (i.e., continuous porosity, ECM-likeness, large surface areas, etc.).

\section{IR and TGA Analysis}

ATR-IR spectra of pristine BNC and CS/BNC composites are shown in Figure 5. Chitosan and cellulose both possess similar chemical structure of repeating D-glucopyranose unites linked with $\beta-1,4$ glycosidic bonds. In the case of pure BNC coat peeled from the fabric-reinforced composite, a sharp characteristic peak of $\mathrm{O}-\mathrm{H}$ stretching appears at $3347.8 \mathrm{~cm}^{-1}$ while the $\mathrm{C}-\mathrm{H}$ stretching appears at $2897 \mathrm{~cm}^{-1}$. Another important characteristic peak of $1660 \mathrm{~cm}^{-1}$ is assigned to the glucose carbonyl stretching (Kim et al., 2011). A series of bands range from 1200 to $1000 \mathrm{~cm}^{-1}$ fingerprint, which are related to the stretching of $\mathrm{C}-\mathrm{O}-\mathrm{C}$ of sugar rings and $\mathrm{C}-\mathrm{O}$ stretching vibrations of the primary (C6) and secondary hydroxyl (C2, C3). For CS/BNC composites, an additional sharp absorption at $1591 \mathrm{~cm}^{-1}$ for $\mathrm{N}-\mathrm{H}$ bending vibrations is distinguished from the cellulose bands, illustrating the CS has been successfully composited with BNC and can retain in the BNC matrix after alkaline purification. And the intensity of peak increases along with the additive concentration rising from 0.25 to $0.75 \%(\mathrm{w} / \mathrm{v}$ ). Compared to the $\mathrm{O}-\mathrm{H}$ stretching peak of $\mathrm{BNC}$ at $3347.8 \mathrm{~cm}^{-1}$, the maximum absorption band of CS/BNC composites shifts to lower wave numbers $\left(3443.0,3342.3,3341.0 \mathrm{~cm}^{-1}\right.$ for $0.25 \% \mathrm{CS} / \mathrm{BNC}, 0.50 \% \mathrm{CS} / \mathrm{BNC}$, and $0.75 \% \mathrm{CS} / \mathrm{BNC}$ respectively),
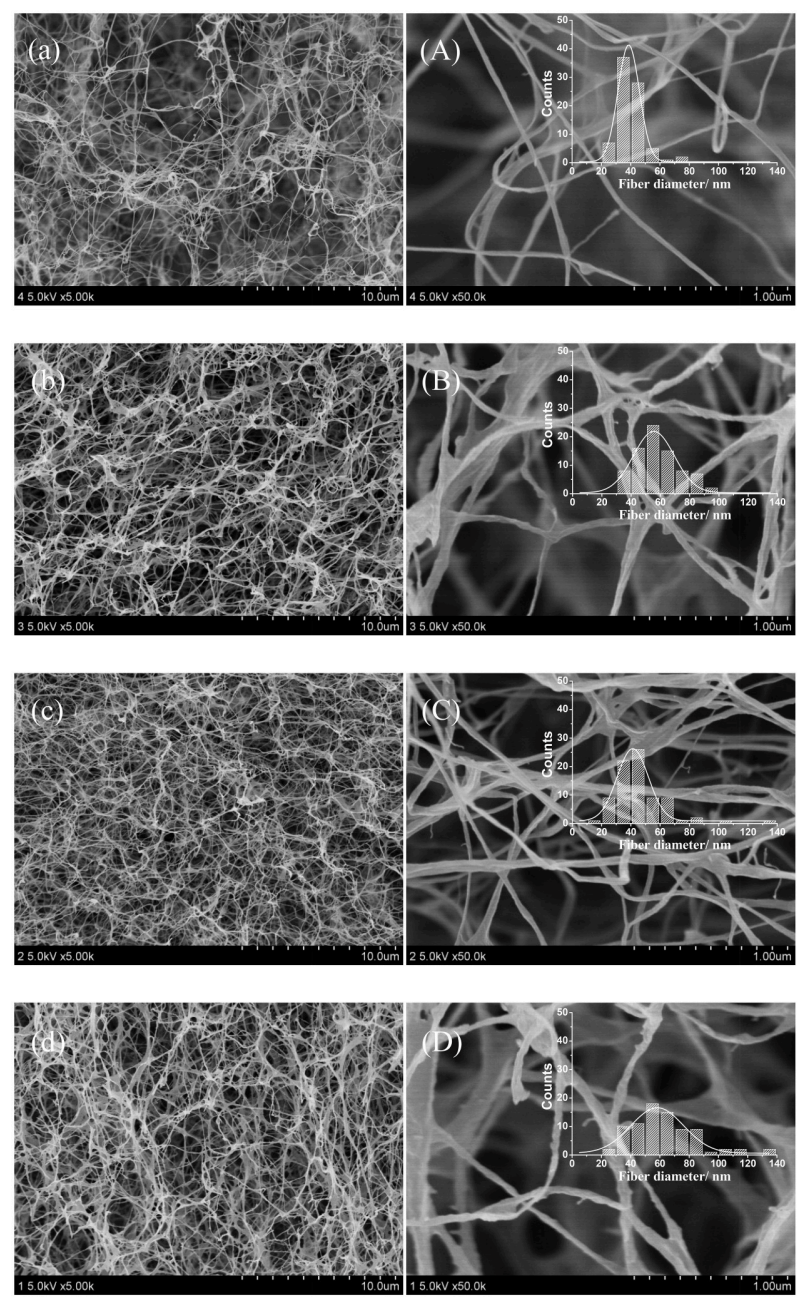

FIGURE 4 | FE-SEM micrographs of the hydrogel coat layer of the reinforced BNC $(a, A)$, the reinforced $0.25 \% C S / B N C(b, B)$, the reinforced $0.50 \% \mathrm{CS} / \mathrm{BNC}(\mathrm{c}, \mathrm{C})$, and the reinforced $0.75 \% \mathrm{CS} / \mathrm{BNC}(\mathrm{d}, \mathrm{D})$ at $\times \mathbf{5 0 0 0}$ and $\times \mathbf{5 0 , 0 0 0}$ magnification, respectively. Embedded small graphs show the diameter distribution of the nanofibers.

implying a decreased degree of hydrogen bonding (Clasen et al., 2006).

Thermo-gravimetric analysis (TGA) was performed with only the hydrogel coat parts of the fabric-reinforced BNC and -reinforced $\mathrm{CS} / \mathrm{BNC}$ composites. Figure $6 \mathrm{~A}$ shows the weight loss of $\mathrm{BNC}, \mathrm{CS}$, and $\mathrm{CS} / \mathrm{BNC}$ vs. temperature in nitrogen atmosphere. The BNC started to degrade from $203^{\circ} \mathrm{C}$ and achieved the maximum decomposition rate at $258.9^{\circ} \mathrm{C}$ as indicated by derivative thermogravimetric curve (DTG) plotted in Figure 6B. Compared to BNC, CS had a slightly higher starting decomposition temperature of $258^{\circ} \mathrm{C}$ and the maximum decomposition rate was recorded at $302.0^{\circ} \mathrm{C}$. CS/BNC composites displayed only one weight loss curve intermediated between that of pure chitosan and pure BNC samples. The DTG plot shows the peaks of decomposition for pure BNC and CS sharply appeared at 258.9 and $302.0^{\circ} \mathrm{C}$, respectively. The broad decomposition rate peaks of CS/BNC composites 

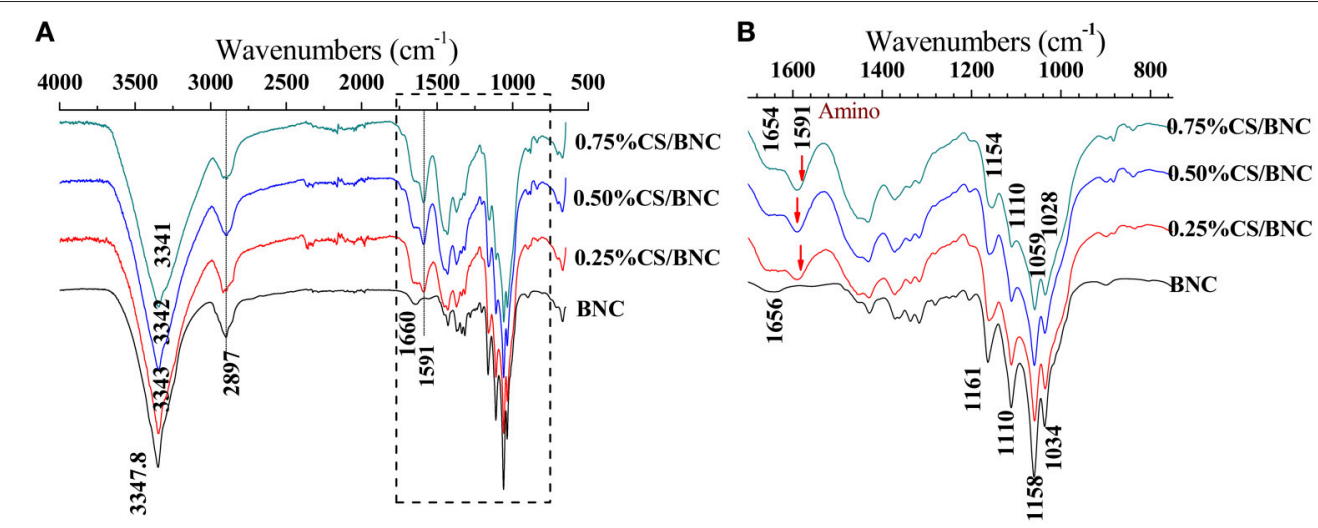

FIGURE 5 | ATR-IR spectra of fabric-reinforced BNC and fabric-reinforced CS/BNC composites: (A) spectra at wavenumber ranging from 4000 to $500 \mathrm{~cm}^{-1}$, (B) spectra are amplified at wavenumber ranging from 1700 to $800 \mathrm{~cm}^{-1}$.

were the results of superposition of the two materials. The maximum decomposition rates of $\mathrm{CS} / \mathrm{BNC}$ composites peaked at $283.1,292.7$, and $298.6^{\circ} \mathrm{C}$ for $0.25 \% \mathrm{CS} / \mathrm{BNC}, 0.50 \% \mathrm{CS} / \mathrm{BNC}$, and $0.75 \% \mathrm{CS} / \mathrm{BNC}$ respectively, showing a rising trend with the addition of CS.

Both the IR and TGA results indicated that CS was wellincorporated with $\mathrm{BNC}$ and generated strong interaction with the cellulose molecules.

\section{Water Holding, Absorption, and Retention Capacity}

The water holding, absorption and retention properties of the integral fabric-reinforced composites and the fabric-removed composite hydrogel coats were investigated separately. As shown in the Table 1, the WHC of the hydrogel coat striped from the reinforced hydrogel composites reached 69.1, 75.6, 84.3, 89.1 g/g for BNC, $0.25 \% \mathrm{CS} / \mathrm{BNC}, 0.50 \% \mathrm{CS} / \mathrm{BNC}$, and $0.75 \% \mathrm{CS} / \mathrm{BNC}$ respectively, indicating the improvement in the WHC along with the increase of chitosan concentration in culture media. However, if measured with the reserved fabric skeleton, the WHC of integral fabric-reinforced hydrogel composites decreased considerably to $18.5,30.0,33.2,45.4 \mathrm{~g} / \mathrm{g}$ for the fabric-reinforced BNC, $0.25 \% \mathrm{CS} / \mathrm{BNC}, 0.50 \% \mathrm{CS} / \mathrm{BNC}$, and $0.75 \% \mathrm{CS} / \mathrm{BNC}$, respectively.

WAC, a parameter reflecting the amount of fluid absorbed by the wound dressing on a basis of gram water per gram mass, is an important feature to absorb wound exudates to maintain a moist physiological and clean environment. The WAC of the freezedried hydrogel coat achieved 56.0, 65.1, 67.2, $78.4 \mathrm{~g} / \mathrm{g}$ for BNC, $0.25 \% \mathrm{CS} / \mathrm{BNC}, 0.50 \% \mathrm{CS} / \mathrm{BNC}$, and $0.75 \% \mathrm{CS} / \mathrm{BNC}$ respectively, which surpassed the naked cotton gauze $(5.7 \mathrm{~g} / \mathrm{g})$ seven-folds and more. The WAC was improved by in situ compositing with CS and improved with increase of CS addition, which is in accordance with a previous report using static cultures (Phisalaphong and Jatupaiboon, 2008). After coating the fabric with nano-fibrillar BNC hydrogel, the overall WAC of the fabricreinforced BNC and -reinforced iCS/BNC $(i=0.25,0.50$, $0.75 \%)$ was improved to a decent level of 19.7, 29.1, 34.1, and $42.7 \mathrm{~g} / \mathrm{g}$, respectively, which is remarkably bigger than $5.7 \mathrm{~g} / \mathrm{g}$ of the cotton gauze fabric. The BNC- and CS/BNC-coating fabric composites should be considered as promising dressing materials.

In order to investigate the fluid distribution of $\mathrm{CS} / \mathrm{BNC}$ composites, a centrifugation method in British Pharmacopeia for alginate wound dressings was performed (Qin, 2004, 2008). The absorbed fluid in dressings can be divided into two kinds: the fluid held between the fibers $\left(\mathrm{W}_{1}-\mathrm{W}_{2}\right)$ and liquid held inside the fibers $\left(\mathrm{W}_{2}-\mathrm{W}_{3}\right)$. The former is more inclined to migrate along textile structure, which could cause macerations of surrounding healthy skin in clinical application, while the later is more stable, which is helpful to maintain an ideal moist healing environment (Qin, 2004, 2008). The ratio of $\left(\mathrm{W}_{1}-\mathrm{W}_{2}\right) /\left(\mathrm{W}_{2}-\right.$ $\mathrm{W}_{3}$ ) reflects the fluid distribution within wound dressings.

Cotton gauze has a hierarchically structural water distribution in the single cotton fiber, multiple yarns, and woven or knitted structure, with a decreasing water mobility (as shown in Figure 7A; Marsh et al., 1953; Qin, 2004). By centrifuging, the spun-out fluid ( $\mathrm{W}_{1}-\mathrm{W}_{2}$, i.e., the fluid held between the fibers) is assigned to those held by meshes of woven or knitted structure (Figure 7Ai) and by interstices in multiple yarns (Figure 7Aii), while those immovable liquid $\left(\mathrm{W}_{2}-\mathrm{W}_{3}\right.$, i.e., the liquid held inside fibers) is supposed to the fluid absorbed into the cell wall of single fiber (Figure 7Aiii). Table 1 shows that the bulk of liquid absorbed into cotton gauze by capillary forces was 5.3 $\mathrm{g} / \mathrm{g}$ as an index of $\left(\mathrm{W}_{1}-\mathrm{W}_{2}\right) / \mathrm{W}_{3}$ and the ratio of $\left(\mathrm{W}_{1}-\right.$ $\left.\mathrm{W}_{2}\right) /\left(\mathrm{W}_{2}-\mathrm{W}_{3}\right)$ was $11.8 \mathrm{~g} / \mathrm{g}$. In contrast, $\mathrm{BNC}$ has a porous nano-fibrillar network constituted by cellulose ribbons of sub$100 \mathrm{~nm}$, therefore the fluid can be held in the nano-porous structure by capillary (Figure 7Biv) and held in the nanofibers (Figure 7Bv) or firmly bound to the plentiful hydroxyl of BNC nanofiber surface (Figure 7Bvi; Klemm et al., 2005). Benefited from the supramolecular structure with high porosity, BNC can absorb abundant liquid within its nanofibril network as the index of $\left(\mathrm{W}_{1}-\mathrm{W}_{2}\right) / \mathrm{W}_{3}$ reached $48.1 \mathrm{~g} / \mathrm{g}$. And the water absorption can be assigned to difficult-to-migrate reaching 7.8 $\mathrm{g} / \mathrm{g}$, which is a magnitude significantly greater than the cotton gauze $(0.4 \mathrm{~g} / \mathrm{g})$ (Table 1$)$. The ratio of $\left(\mathrm{W}_{1}-\mathrm{W}_{2}\right) /\left(\mathrm{W}_{2}-\mathrm{W}_{3}\right)$ of $\mathrm{BNC}$ was detected to $6.2 \mathrm{~g} / \mathrm{g}$, indicating water retention ability of $\mathrm{BNC}$ is better than cotton gauze and this performance is 

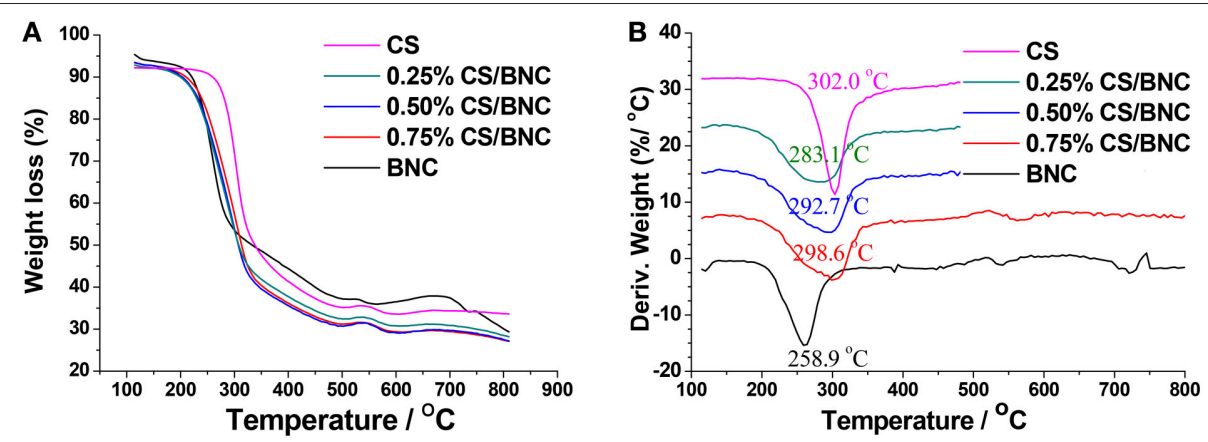

FIGURE 6 | Typical thermal degradation profiles (A) and derivative curves (B) for the hydrogel coat of fabric-reinforced BNC and -reinforced CS/BNC composites.

TABLE 1 | Water holding capacity and absorption behavior of naked cotton gauze, integrated reinforced CS/BNC composites and their separated hydrogel coats only.

\begin{tabular}{|c|c|c|c|c|c|}
\hline Sample & $W H C^{*}(g / g)$ & WAC $^{*}(g / g)$ & $\left(W_{1}-W_{2}\right) / W_{3}^{*}(g / g)$ & $\left(W_{2}-W_{3}\right) / W_{3}^{*}(g / g)$ & $\left(W_{1}-W_{2}\right) /\left(W_{2}-W_{3}\right)^{*}(g / g)$ \\
\hline Cotton gauze & Null & $5.7 \pm 0.3$ & $5.3 \pm 0.5$ & $0.4 \pm 0.1$ & $11.8 \pm 0.6$ \\
\hline BNC (coat) & $69.1 \pm 5.1$ & $56.0 \pm 3.8$ & $48.1 \pm 3.6$ & $7.8 \pm 0.1$ & $6.2 \pm 0.5$ \\
\hline $0.50 \% \mathrm{CS} / \mathrm{BNC}$ (coat) & $84.3 \pm 6.4$ & $67.2 \pm 3.2$ & $59.0 \pm 3.1$ & $8.2 \pm 0.3$ & $7.2 \pm 0.5$ \\
\hline 0.75\%CS/BNC (coat) & $89.1 \pm 7.3$ & $78.4 \pm 5.2$ & $69.0 \pm 6.2$ & $9.5 \pm 1.1$ & $7.1 \pm 0.5$ \\
\hline 0.50\%CS/BNC (integrated) & $33.2 \pm 1.6$ & $34.1 \pm 1.6$ & $30.2 \pm 2.2$ & $3.9 \pm 0.2$ & $7.7 \pm 0.4$ \\
\hline $0.75 \% \mathrm{CS} / \mathrm{BNC}$ (integrated) & $45.4 \pm 2.2$ & $42.7 \pm 3.3$ & $38.0 \pm 2.8$ & $4.8 \pm 0.5$ & $8.0 \pm 0.2$ \\
\hline
\end{tabular}

"Each test was repeated eight times, and the means \pm standard deviations were given.

close to some commercial alginate dressings, for instance, a Curasorb $^{\mathrm{TM}}$ dressing had $32.0 \mathrm{~g} / \mathrm{g}$ of $\left(\mathrm{W}_{1}-\mathrm{W}_{2}\right) / \mathrm{W}_{3}$ and 8.62 $\mathrm{g} / \mathrm{g}$ of $\left(\mathrm{W}_{1}-\mathrm{W}_{2}\right) /\left(\mathrm{W}_{2}-\mathrm{W}_{3}\right)$ (Qin, 2004). After compositing with $\mathrm{CS}$, both $\left(\mathrm{W}_{1}-\mathrm{W}_{2}\right) / \mathrm{W}_{3}$ and $\left(\mathrm{W}_{2}-\mathrm{W}_{3}\right) / \mathrm{W}_{3}$ of the BNC hydrogel coat were improved, indicating the hydrophilic CS polymers improve both the structural water absorption capacity and the ability to bound water. The ratios of $\left(\mathrm{W}_{1}-\mathrm{W}_{2}\right) /\left(\mathrm{W}_{2}-\right.$ $\mathrm{W}_{3}$ ) of CS/BNC composites were $7.3,7.2$, and $7.1 \mathrm{~g} / \mathrm{g}$ for $0.25 \% \mathrm{CS} / \mathrm{BNC}, 0.50 \% \mathrm{CS} / \mathrm{BNC}$, and $0.75 \% \mathrm{CS} / \mathrm{BNC}$ respectively, which are slightly higher than $6.2 \mathrm{~g} / \mathrm{g}$ of BNC. As a whole material, Table 1 shows the ratio of $\left(\mathrm{W}_{1}-\mathrm{W}_{2}\right) /\left(\mathrm{W}_{2}-\mathrm{W}_{3}\right)$ for fabric-reinforced BNC and iCS/BNC $(i=0.25,0.50,0.75 \%)$ were $6.9,8.4,7.7,8.0 \mathrm{~g} / \mathrm{g}$ respectively, which indicates the overall liquid retention behaviors of the fabric-reinforced $\mathrm{BNC}$ and CS/BNC composites are near to the corresponding hydrogel coats. This is because the hydrogel coats performed the majority of the absorption.

\section{Mechanical Properties}

The tensile mechanical properties of the fabric-reinforced BNC and fabric-reinforced CS/BNC composites are shown in Figure 8 in comparison with the uncoated cotton gauze and pristine $\mathrm{BNC}$ sheets from a $10-\mathrm{d}$ static culture. The ultimate tensile stress of the high water swollen pristine BNC hydrogel sheets from static culture was only $0.03 \pm 0.04 \mathrm{MPa}$, which is apparently far weaker than ordinary fabrics (in this case cotton gauze showed 9.0 $\pm 0.3 \mathrm{MPa}$, Figure $\mathbf{8 B}$ ). The ultimate tensile stress of the fabric-reinforced BNC and -reinforced CS/BNC composites ranged from 1.4 to $1.5 \mathrm{MPa}$ (Figure $8 \mathrm{~B}$ ) and no significant difference was found among them. These relatively low values should be attributed to the remarkable thickness of the fabricreinforced $\mathrm{BNC}$ and $\mathrm{CS} / \mathrm{BNC}$ composites since the tensile stress $(\mathrm{MPa})$ is a normalized value from tensile force $(\mathrm{N})$ through dividing by the cross-sectional area $\left(\mathrm{m}^{2}\right)$ of the material. Figure 8C shows that the fabric skeletons strengthen the hydrogel obviously, resulting in immediately boosting in tensile fracture strength. Not surprisingly, with similar hydrogel thickness, the fabric-reinforced BNC and $\mathrm{iCS} / \mathrm{BNC}(i=0.25,0.50,0.75 \%)$ obtained similar ultimate tensile stress values. However, the tensile fracture force of reinforced BNC hydrogel or reinforced CS/BNC composites all surpassed $36 \mathrm{~N}$ (Figure 8C), which was significantly higher than the uncoated cotton gauze (29.3 $\mathrm{N})$. The results imply a composite effect that the BNC or $\mathrm{CS} / \mathrm{BNC}$ hydrogel coat could reinforce the fabric skeleton as well. Noteworthily, the increments in fracture force values were still far higher than the pristine BNC hydrogel sheets without fabric skeleton $(0.8 \mathrm{~N})$. This indicates the increments in facture force should be mainly attributed to the cohesion between the hydrogel coats and the fabrics rather than the hydrogel coats themselves. BNC nano-filaments attachment on the surface of 

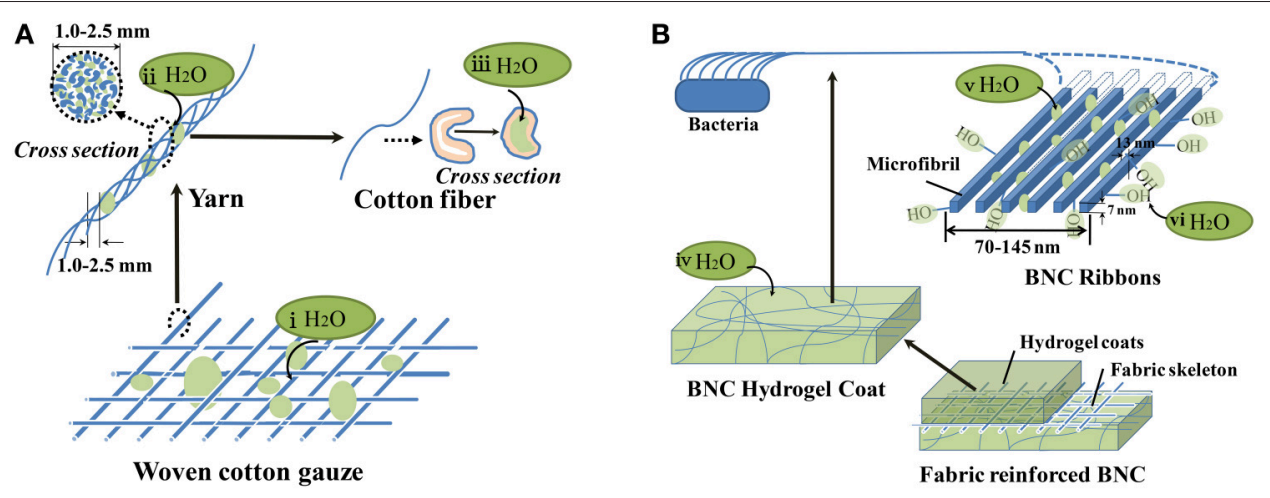

FIGURE 7 | Hypothesized absorption distribution in the woven cotton gauze (A) and BNC hydrogel (B): water holding in the meshes of warp and weft (i); water retained in crevices of yarns (ii); water absorbed into cotton fibers (iii); water retained in the nano-fibrillar networks (iv); water interred BNC nano-fiber (v); water bounded to BNC nano-ribbons (vi).
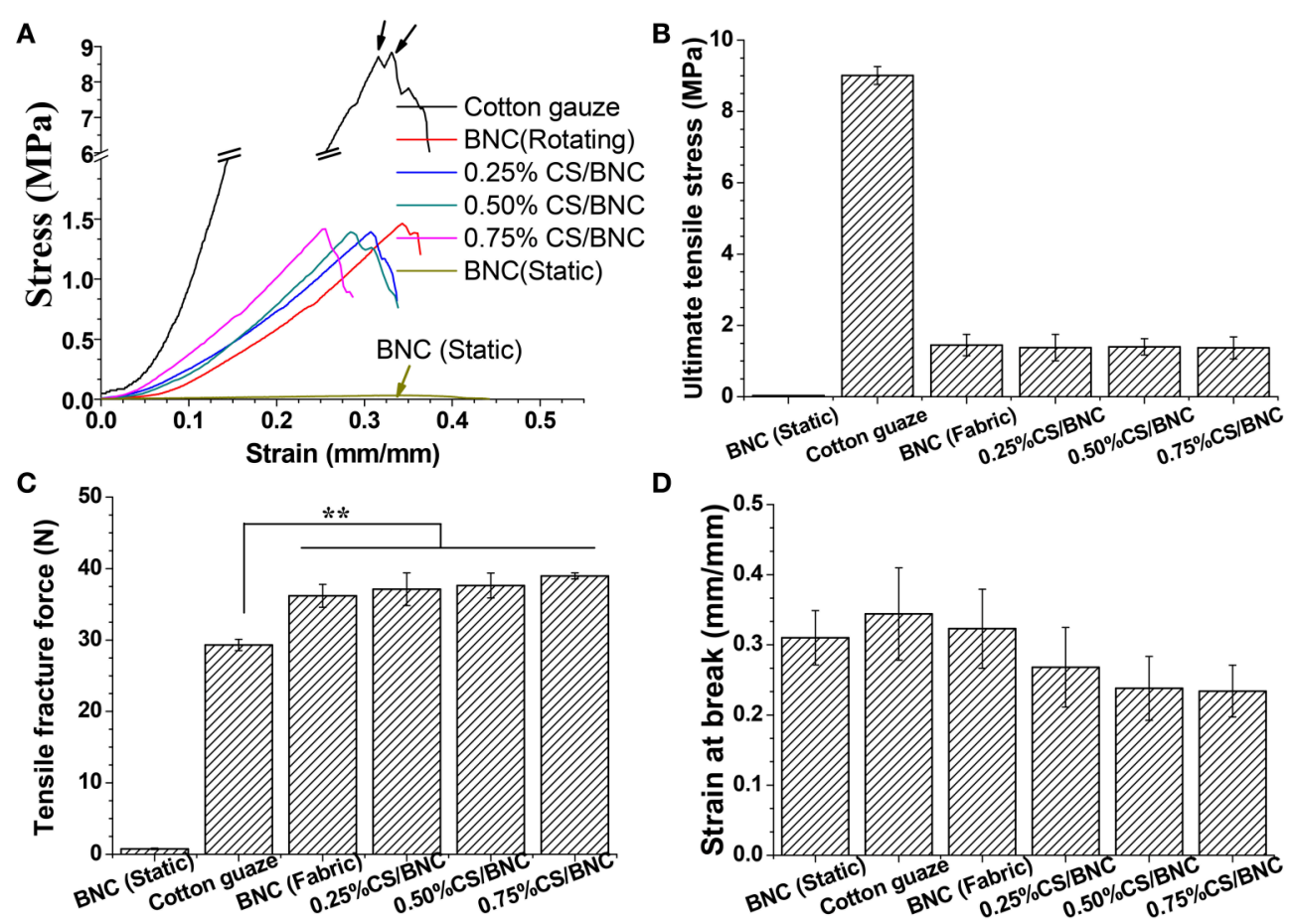

FIGURE 8 | Tensile properties of the pure BNC sheet (static), naked cotton gauze, fabric-reinforced BNC (rotating), and fabric-reinforced CS/BNC composites: (A) representative strain-stress curves, (B) ultimate tensile stress, (C) tensile fracture force, (D) strain at break. The tests were performed in octuplicate, and means \pm standard deviations were given.

natural hemp fibers was found to greatly improve the interfacial shear strength of fibers, which creates strong viscous friction on fiber surface (Pommet et al., 2008). Therefore, the BNC attachments would enhance the interfacial adhesion among cotton fibers in each individual yarns, as well as enhance the interfacial adhesion between warp and weft yarns, by which the cotton gauzes got more integrated and reinforced. The cohesive effects could decrease the interfacial displacement in inter- or inner- yarns, for which the values of strain at break of fabric-reinforced BNC $(0.32 \mathrm{~mm} / \mathrm{mm})$ and -reinforced CS/BNCs $(0.23$ to $0.27 \mathrm{~mm} / \mathrm{mm})$ were slightly lower than the uncoated cotton gauze $(0.34 \mathrm{~mm} / \mathrm{mm}$, as shown in Figure 8D). The cotton gauze fractured in the form that yarns broke one by one, resulting in the multiple peaks in the stress-strain curve as indicated by black arrows in Figure 8A. But after coating with $\mathrm{BNC}$ or CS/BNC hydrogel, it fractured almost simultaneously at all stretched yarns (reflected by the single-peaked stress-strain curves in Figure 8A). It also indicates an improvement in the 
integration between yarns. The results suggest that the inner frame of fabric can be introduced into BNC hydrogel to augment its tensile property, and the fabric itself also can be reinforced by BNC coating for more intense bonding strength in inter- and inner-yarns.

\section{Antibacterial Ability}

Antibacterial ability of the reinforced-CS/BNC in both hydrogel state and lyophilized state was investigated by using two methods: absorption method and shake-flask method (saline). The results of antimicrobial assessment are shown in Figure 9 and Table 2.

By using the absorption method (Figure 2A), the bacterial inocula directly touched with control and test samples. For the lyophilized samples, the number of bacterial colonies obtained from all the CS/BNC composites was significantly lower than the BNC control group after $24 \mathrm{~h}$ contact (Figure 9a). The bacterial reduction percentage ranged from 97.79 to $99.24 \%$ with a corresponding $A$-value ranging from 1.65 to 2.12 for S. aureus, and from 96.67 to $99.76 \%$ with a corresponding $A$ value ranging from 1.46 to 2.59 for $E$. coli, respectively (Table 2), demonstrating a positive broad-spectrum antibacterial effect. In contrast with the BNC $(24 \mathrm{~h})$, the bacterial growth on $\mathrm{CS} / \mathrm{BNC}$ composites was significantly lower (decreased more than 1 logarithmic value, as shown in Figure 9a), showing a remarkable inhibition on bacterial growth (bacteriostatic activity). However, as compared with the bacterial growth after just inoculation (BNC $0 \mathrm{~h}$ ), the bacterial growth on CS/BNC composites after $24 \mathrm{~h}$ contact only had a small reduction (less than 1 logarithmic value), even though slightly increased for $0.25 \%$ CS/BNC, exhibiting almost no bactericidal ability. This result suggests that the lyophilized CS/BNC composites mainly exerted bacteriostatic effect (inhibition function on growth) instead of bactericidal effect. This result is in accordance with previous literatures reporting that the antimicrobial activity of CS is bacteriostatic rather than bactericidal (Raafat et al., 2008; Kong et al., 2010). For the gelatinous samples (Figure 9b), the bacterial numbers on all CS/BNC composites were lower than the BNC group at $24 \mathrm{~h}$, but higher than the number after just inoculation (BNC $0 \mathrm{~h}$ ), also showing a bacteriostatic ability. The antibacterial effect of hydrogel samples of $0.25 \% \mathrm{CS} / \mathrm{BNC}$, $0.50 \% \mathrm{CS} / \mathrm{BNC}$, and $0.75 \% \mathrm{CS} / \mathrm{BNC}$ increased with the addition of CS and the bacterial reduction percentage was 29.71, 73.22, $88.37 \%$ against $S$. aureus, and 27.36, 69.26, 85.98\% against E. coli, respectively (Table 2). Taking into account the above results it is suggested that the antibacterial effect of lyophilized samples was much stronger than that of the corresponding hydrogel samples.

A modified shake-flask method (Singh et al., 2012) was also applied (Figure 2B) on the basis of the standard dynamic (a)

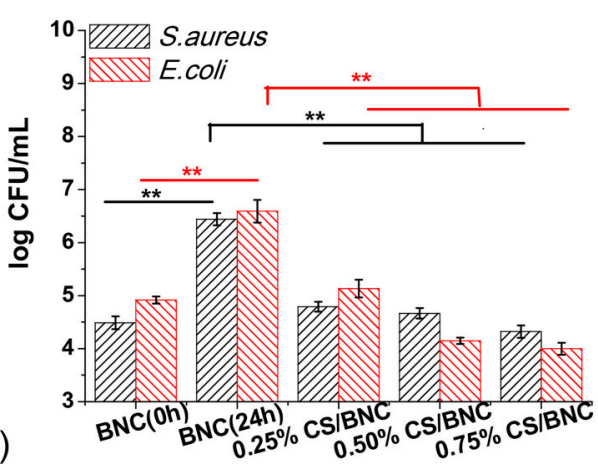

(A)

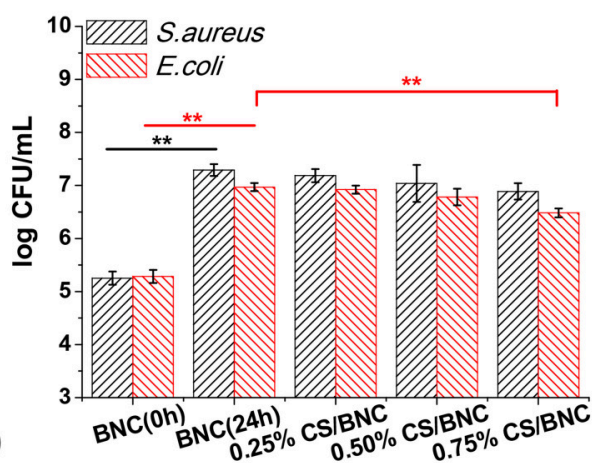

(b)

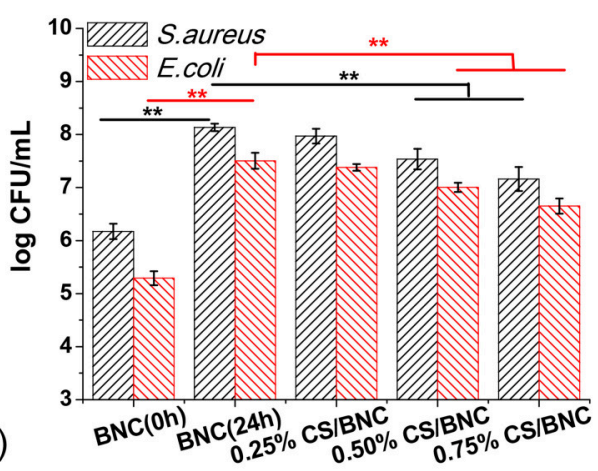

(B)

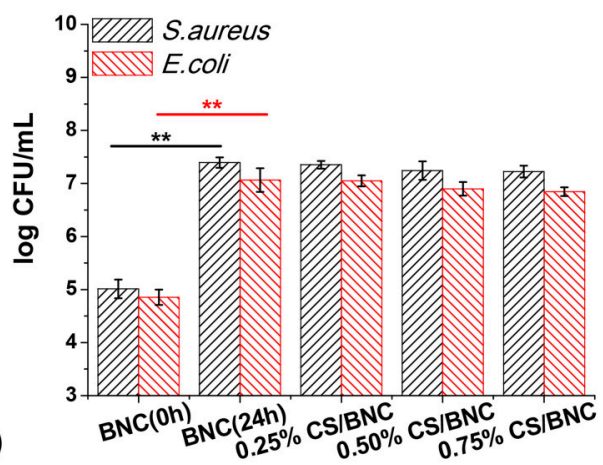

FIGURE 9 | Antibacterial activity of the fabric-reinforced CS/BNC composites in two different states against. S. aureus and $E$. coli using two different methods: (a) lyophilized samples and (b) hydrogel samples were evaluated with the absorption method in $24 \mathrm{~h}$ touching, and (A) lyophilized samples and (B) hydrogel samples were evaluated with the shake-flask method in $24 \mathrm{~h}$ shaking incubation. The experiments were performed in triplicate, and the logarithmic mean values of bacterial colonies and standard deviations were given. 
TABLE 2 | The percentage growth reduction (R) and antibacterial activity (A) of antibacterial tests for fabric-reinforced composites.

\begin{tabular}{|c|c|c|c|c|c|c|c|c|c|}
\hline \multirow[t]{3}{*}{ State } & \multirow[t]{3}{*}{ Samples } & \multicolumn{4}{|c|}{ Absorption method } & \multicolumn{4}{|c|}{ Shake-flask method } \\
\hline & & \multicolumn{2}{|c|}{ S. aureus } & \multicolumn{2}{|c|}{ E. coli } & \multicolumn{2}{|c|}{ S. aureus } & \multicolumn{2}{|c|}{ E. coli } \\
\hline & & R (\%) & $A^{*}$ & R (\%) & $A^{*}$ & R (\%) & $A^{*}$ & R (\%) & $A^{*}$ \\
\hline \multirow[t]{3}{*}{ Lyophilized } & $0.25 \% \mathrm{CS} / \mathrm{BNC}$ & 97.79 & 1.65 & 96.67 & 1.46 & 16.07 & 0.08 & 1.32 & 0.05 \\
\hline & $0.50 \% \mathrm{CS} / \mathrm{BNC}$ & 98.34 & 1.77 & 99.67 & 2.44 & 27.36 & 0.22 & 32.62 & 0.19 \\
\hline & $0.75 \% \mathrm{CS} / \mathrm{BNC}$ & 99.24 & 2.12 & 99.76 & 2.59 & 56.97 & 0.38 & 67.24 & 0.49 \\
\hline \multirow[t]{3}{*}{ Hydrogel } & $0.25 \% \mathrm{CS} / \mathrm{BNC}$ & 29.71 & 0.16 & 27.36 & 0.12 & 9.89 & 0.04 & 10.35 & 0.01 \\
\hline & $0.50 \% \mathrm{CS} / \mathrm{BNC}$ & 73.22 & 0.60 & 69.26 & 0.50 & 26.21 & 0.15 & 36.13 & 0.16 \\
\hline & $0.75 \% \mathrm{CS} / \mathrm{BNC}$ & 88.37 & 0.96 & 85.98 & 0.85 & 32.05 & 0.17 & 44.53 & 0.22 \\
\hline
\end{tabular}

*The growth value on the control sample (F-value) for all the tests was more than 1.5, which verifies the effectiveness of antibacterial tests.

shake-flask method (ASTM-E2149, 2010), where PBS was used instead of nutrient broth and the number of bacterial colonies were determined by a plate counting method. The results are shown in Figures 9A,B. Without nutrient addition, the $\mathrm{F}$ value showed a significant bacterial growth corresponding to 2.01 and 1.68 with the freeze-dried control samples, and showed a significant bacterial growth corresponding to 2.38 and 2.21 with the hydrogel control samples against $S$. aureus and E. coli, respectively (Table 2). Figures $\mathbf{9 A , B}$ show the number of viable bacterial cells in the CS/BNC-added buffer solution was slightly decreased as compared to the bacterial population of BNC (24 h) group. However, both the lyophilized samples and the gelatinous samples exhibited very weak bacteriostatic effect in the dynamic touch because the percentage of microbial reduction for majority test groups was less than $50 \%$ against both S. aureus and E. coli (as shown in Table 2). However, the lyophilized 0.75\%CS/BNC showed a relatively significant reduction against $S$. aureus and E. coli in viable bacterial counts in comparison to BNC (24 h), corresponding to a percentage growth reduction of 56.97 and $67.24 \%$, respectively (as shown in Table 2 ).

In addition to the two quantitative tests, a semi-quantitative test with the agar plate diffusion method was also implemented to evaluate both hydrogel and lyophilized samples. However, no visible inhibition zones have been observed in all the CS/BNC samples for both bacterial species.

The results showed the antibacterial activity of reinforced $\mathrm{CS} / \mathrm{BNC}$ composites had great discrepancies among the different evaluation methods and also among different sample's physical states. Antibacterial activity was more remarkable in lyophilized state rather than hydrogel state, and was effectively detected in absorption method rather than in shake-flask method. This is because the antibacterial effect of CS is dose-dependent (Raafat et al., 2008), and is also heavily influenced by physical states and solid shapes (Kong et al., 2010). Although the CS concentration in culture media was very low $(0.25$ to $0.75 \%$, $\mathrm{w} / \mathrm{v})$ during in situ bio-synthesis of CS/BNC composites, CS proportion was able to reach as high as approximate $40 \%$ in the dehydrated CS/BNC matrix. This makes internal space of $\mathrm{CS} / \mathrm{BNC}$ matrix be of regionally high CS content, which forms a strong bacteriostatic micro-environment. The bacterial growth was strongly inhibited by long-time exposure to the local CS-rich matrix in the absorption method. However, in the shake-flask method, the regionally CS-rich CS/BNC matrix could not affect the bacterial growth of surrounding bacterial suspension in overall only by means of the repeatedly and instantly contacting with the CS/BNC of a standard addition amount $(0.01 \mathrm{~g} / \mathrm{mL})$. The results showed that the bacteriostatic effect of CS/BNC composites was confined within the matrix only, instead of spreading around. The lyophilized sponge-like composites are able to rapidly absorb the water of the droplets of liquid bacterial inocula into internal matrix in the absorption method, while the liquid inocula should only be spread on the superficial surface of hydrogel samples after dropping since the hydrogel samples are saturated with water. The water absorption into the lyophilized samples makes more bacterial cells exposed to or contacted with the CS/BNC matrix, resulting in a better bacteriostatic effect than the gelatinous samples. From another point of view, the practical CS proportion in the gelatinous samples was much lower than that in the lyophilized samples by taking into account water proportion, which should lead to more survival cells. In agar diffusion tests, no inhibition zone was apparent for all the samples, reflecting the bacteriostatic effect of CS/BNC is only confined within the matrix.

\section{CONCLUSIONS}

Fabric-reinforced CS/BNC composite sheets were prepared successfully through the in situ rotating culture technology, and the inherent structural characteristics of BNC hydrogel coat were preserved. The fabric skeleton sustained most of the tensile force, which directly boosts the tensile strength. The fabrics functioned as the supports of cellulosic biofilm immobilization in rotating cultures. This rotating culture process not only has higher productivity than static culture, but also reduces the inhibition effects of the CS on G. xylinus growth and cellulose production. CS dispersed in culture media could be incorporated into BNC matrix successfully with this rotating culture technology, and could be retained in the hydrogel substrate after the rigorous alkaline purification process. Addition of CS into the culture media modified the nano-fibrillar networks of BNC and improved valuable features including water absorbing and 
maintaining properties. The CS/BNC composite sheets showed a good bacteriostatic effect, particularly in the lyophilized sponge state. These features endow the fabric-reinforced CS/BNC composites with great potential as excellent medical materials for wound dressings. Furthermore, the proposed in situ rotating culture technology could be used to integrate other materials including particles and soluble polymers into the nanofibril network of BNC to generate new composites.

\section{AUTHOR CONTRIBUTIONS}

FFH designed and coordinated the study and revised the manuscript. PZ and LC contributed the preparation of the manuscript. PZ carried out most of the experiments and analyzed

\section{REFERENCES}

Agrawal, A., Rahbar, N., and Calvert, P. D. (2013). Strong fiber-reinforced hydrogel. Acta Biomater. 9, 5313-5318. doi: 10.1016/j.actbio.2012.10.011

Bäckdahl, H., Helenius, G., Bodin, A., Nannmark, U., Johansson, B. R., Risberg, B., et al. (2006). Mechanical properties of bacterial cellulose and interactions with smooth muscle cells. Biomaterials 27, 2141-2149. doi: 10.1016/j.biomaterials.2005.10.026

Bäckdahl, H., Risberg, B., and Gatenholm, P. (2011). Observations on bacterial cellulose tube formation for application as vascular graft. Mater. Sci. Eng. C Mater. Biol. Appl. 31, 14-21. doi: 10.1016/j.msec.2010.07.010

Bodin, A., Bharadwaj, S., Wu, S., Gatenholm, P., Atala, A., and Zhang, Y. (2010). Tissue-engineered conduit using urine-derived stem cells seeded bacterial cellulose polymer in urinary reconstruction and diversion. Biomaterials 31, 8889-8901. doi: 10.1016/j.biomaterials.2010.07.108

Brown, E., Zhang, J., and Laborie, M.-P. (2011). Never-dried bacterial cellulose/fibrin composites: preparation, morphology and mechanical properties. Cellulose 18, 631-641. doi: 10.1007/s10570-011-9500-8

Cai, Z., and Kim, J. (2010). Bacterial cellulose/poly(ethylene glycol) composite: characterization and first evaluation of biocompatibility. Cellulose 17, 83-91. doi: 10.1007/s10570-009-9362-5

Chen, H. H., Chen, L. C., Huang, H. C., and Lin, S. B. (2011). In situ modification of bacterial cellulose nanostructure by adding CMC during the growth of Gluconacetobacter xylinus. Cellulose 18, 1573-1583. doi: 10.1007/s10570-0119594-z

Cheng, K. C., Catchmark, J., and Demirci, A. (2009). Enhanced production of bacterial cellulose by using a biofilm reactor and its material property analysis. J. Biol. Eng. 3:12. doi: 10.1186/1754-1611-3-12

Ciechańska, D. (2004). Multifunctional bacterial cellulose/chitosan composite materials for medical applications. Fibers Text. East. Eur. 12, 69-72. Available online at: https://www.infona.pl/resource/bwmeta1.element.baztechc326df6d-d738-4e23-ad3b-7157813ebde9/tab/summary

Clasen, C., Sultanova, B., Wilhelms, T., Heisig, P., and Kulicke, W. M. (2006). Effects of different drying processes on the material properties of bacterial cellulose membranes. Macromol. Symp. 244, 48-58. doi: 10.1002/masy.200651204

Czaja, W., Krystynowicz, A., Bielecki, S., and Brown, J. R. M. (2006). Microbial cellulose-the natural power to heal wounds. Biomaterials 27, 145-151. doi: 10.1016/j.biomaterials.2005.07.035

Czaja, W., Krystynowicz, A., Kawecki, M., Wysota, K., Sakiel, S., Wróblewski, P., et al. (2007). "Biomedical applications of microbial cellulose in burn wound recovery," in Cellulose: Molecular and Structural Biology, eds R. Brown and J. Saxena (Houten: Springer), 307-321.

Fernandes, S. C. M., Oliveira, L., Freire, C. S. R., Silvestre, A. J. D., Neto, C. P., Gandini, A., et al. (2009). Novel transparent nanocomposite films based on chitosan and bacterial cellulose. Green Chem. 11, 2023-2029. doi: $10.1039 / \mathrm{b} 919112 \mathrm{~g}$ the results. QZ performed some antibacterial tests. All authors read and approved the final manuscript.

\section{ACKNOWLEDGMENTS}

Financial supports provided by Program for New Century Excellent Talents in University (NCET-12-0828), by the National Natural Science Foundation of China (51373031), the Science and Technology Commission of Shanghai Municipality (15520720800), the 111 Project B07024, the Fundamental Research Funds for the Central Universities (2232014A304), and Innovation Foundation of Donghua University for Doctoral Candidates (CUSF-DH-D-2013041) are gratefully acknowledged.

Fontana, J. D., Souza, A. M., Fontana, C. K., Torriani, I. L., Moreschi, J. C., Gallotti, B. J., et al. (1990). Acetobacter cellulose pellicle as a temporary skin substitute. Appl. Biochem. Biotechnol. 24-25, 253-264. doi: 10.1007/BF02 920250

Fu, L., Zhang, J., and Yang, G. (2013). Present status and applications of bacterial cellulose-based materials for skin tissue repair. Carbohydr. Polym. 92, 1432-1442. doi: 10.1016/j.carbpol.2012.10.071

Fu, L., Zhang, Y., Li, C., Wu, Z., Zhuo, Q., Huang, X., et al. (2012). Skin tissue repair materials from bacterial cellulose by a multilayer fermentation method. J. Mater. Chem. 22, 12349-12357. doi: 10.1039/C2JM00134A

Galván Márquez, I., Akuaku, J., Cruz, I., Cheetham, J., Golshani, A., and Smith, M. L. (2013). Disruption of protein synthesis as antifungal mode of action by chitosan. Int. J. Food Microbiol. 164, 108-112. doi: 10.1016/j.ijfoodmicro.2013.03.025

Gatenholm, P., and Klemm, D. (2010). Bacterial nanocellulose as a renewable material for biomedical applications. MRS Bull. 35, 208-213. doi: $10.1557 / \mathrm{mrs} 2010.653$

Hagiwara, Y., Putra, A., Kakugo, A., Furukawa, H., and Gong, J. (2010). Ligamentlike tough double-network hydrogel based on bacterial cellulose. Cellulose 17, 93-101. doi: 10.1007/s10570-009-9357-2

Heßler, N., and Klemm, D. (2009). Alteration of bacterial nanocellulose structure by in situ modification using polyethylene glycol and carbohydrate additives. Cellulose 16, 899-910. doi: 10.1007/s10570-009-9301-5

Hong, F. (2012b). Method for Preparing Nano-Cellulose Antibacterial Composite Material through On-Line Culture. China, Patent CN102552965/CN2012114527. Available online at: http://worldwide. espacenet.com/publicationDetails/biblio? $\mathrm{DB}=\mathrm{EPODOC} \& \mathrm{II}=0 \& \mathrm{ND}=3 \&$ adjacent $=$ true $\&$ locale $=$ en_EP\&FT $=$ D\&date $=20120711 \& C C=C N \& N R=$ $102552965 \mathrm{~A} \& \mathrm{KC}=\mathrm{A}$

Hong, F., Tang, S., and Yang, X. (2012a). Device for Quickly Preparing Bacterial Cellulose Composite Material in Large Scale. China, Patent CN202830006/CN2012221360U. Available online at: http://worldwide. espacenet.com/publicationDetails/biblio? $\mathrm{DB}=\mathrm{EPODOC} \& \mathrm{II}=0 \& \mathrm{ND}=3 \&$ adjacent $=$ true\&locale $=$ en_EP\&FT $=$ D\&date $=20130327 \& C C=C N \& N R=$ $202830006 \mathrm{U} \& \mathrm{KC}=\mathrm{U}$

Hong, F., Wei, B., and Chen, L. (2015). Preliminary study on biosynthesis of bacterial nanocellulose tubes in a novel double-silicone-tube bioreactor for potential vascular prosthesis. Biomed. Res. Int. 9:560365. doi: $10.1155 / 2015 / 560365$

Hornung, M., Ludwig, M., Gerrard, A., and Schmauder, H. P. (2006). Optimizing the production of bacterial cellulose in surface culture: evaluation of substrate mass transfer influences on the bioreaction (Part 1). Eng. Life Sci. 6, 537-545. doi: $10.1002 /$ elsc.200620162

Kim, J., Cai, Z., Lee, H. S., Choi, G. S., Lee, D. H., and Jo, C. (2011). Preparation and characterization of a Bacterial cellulose/chitosan composite for potential biomedical application. J. Polym. Res. 18, 739-744. doi: 10.1007/s10965-0109470-9 
Klemm, D., Heublein, B., Fink, H.-P., and Bohn, A. (2005). Cellulose: fascinating biopolymer and sustainable raw material. Angew. Chem. Int. Edit. 44, 3358-3393. doi: 10.1002/anie.200460587

Kong, M., Chen, X. G., Xing, K., and Park, H. J. (2010). Antimicrobial properties of chitosan and mode of action: a state of the art review. Int. J. Food Microbiol. 144, 51-63. doi: 10.1016/j.ijfoodmicro.2010.09.012

Lin, S. B., Hsu, C.-P., Chen, L.-C., and Chen, H.-H. (2009). Adding enzymatically modified gelatin to enhance the rehydration abilities and mechanical properties of bacterial cellulose. Food Hydrocolloids 23, 2195-2203. doi: 10.1016/j.foodhyd.2009.05.011

Lin, W. C., Lien, C. C., Yeh, H. J., Yu, C. M., and Hsu, S. H. (2013). Bacterial cellulose and bacterial cellulose-chitosan membranes for wound dressing applications. Carbohydr. Polym. 94, 603-611. doi: 10.1016/j.carbpol.2013.01.076

Lopes, J. L., Machado, J. M., Castanheira, L., Granja, P. L., Gama, F. M., Dourado, F., et al. (2011). Friction and wear behaviour of bacterial cellulose against articular cartilage. Wear 271, 2328-2333. doi: 10.1016/j.wear.2010.12.042

Maksimova, Y. G. (2014). Microbial biofilms in biotechnological processes. Appl. Biochem. Microbiol. 50, 750-760. doi: 10.1134/S0003683814080043

Marsh, P. B., Merola, G. V., and Simpson, M. E. (1953). Experiments with an alkali swelling-centrifuge test applied to cotton fiber. Text. Res. J. 23, 831-841. doi: 10.1177/004051755302301111

Martínez Ávila, H., Schwarz, S., Feldmann, E. M., Mantas, A., von Bomhard, A., Gatenholm, P., et al. (2014). Biocompatibility evaluation of densified bacterial nanocellulose hydrogel as an implant material for auricular cartilage regeneration. Appl. Microbiol. Biotechnol. 98, 7423-7435. doi: 10.1007/s00253014-5819-z

McKenna, B., Mikkelsen, D., Wehr, J., Gidley, M., and Menzies, N. (2009). Mechanical and structural properties of native and alkali-treated bacterial cellulose produced by Gluconacetobacter xylinus strain ATCC 53524. Cellulose 16, 1047-1055. doi: 10.1007/s10570-009-9340-y

Meftahi, A., Khajavi, R., Rashidi, A., Sattari, M., Yazdanshenas, M., and Torabi, M. (2010). The effects of cotton gauze coating with microbial cellulose. Cellulose 17, 199-204. doi: 10.1007/s10570-009-9377-y

Nakayama, A., Kakugo, A., Gong, J. P., Osada, Y., Takai, M., Erata, T., et al. (2004). High mechanical strength double-network hydrogel with bacterial cellulose. Adv. Funct.Mater. 14, 1124-1128. doi: 10.1002/adfm.200305197

Nge, T., Nogi, M., Yano, H., and Sugiyama, J. (2010). Microstructure and mechanical properties of bacterial cellulose/chitosan porous scaffold. Cellulose 17, 349-363. doi: 10.1007/s10570-009-9394-x

Petersen, N., and Gatenholm, P. (2011). Bacterial cellulose-based materials and medical devices: current state and perspectives. App. Microbiol. Biotechnol. 91, 1277-1286. doi: 10.1007/s00253-011-3432-y

Phisalaphong, M., and Jatupaiboon, N. (2008). Biosynthesis and characterization of bacteria cellulose-chitosan film. Carbohydr. Polym. 74, 482-488. doi: 10.1016/j.carbpol.2008.04.004

Pommet, M., Juntaro, J., Heng, J. Y. Y., Mantalaris, A., Lee, A. F., Wilson, K., et al. (2008). Surface modification of natural fibers using bacteria: depositing bacterial cellulose onto natural fibers to create hierarchical fiber reinforced nanocomposites. Biomacromolecules 9, 1643-1651. doi: 10.1021/bm800169g

Qin, Y. (2004). Absorption characteristics of alginate wound dressings. J. Appl. Polym. Sci. 91, 953-957. doi: 10.1002/app.13291

Qin, Y. (2008). The gel swelling properties of alginate fibers and their applications in wound management. Polym. Adv. Technol. 19, 6-14. doi: 10.1002/pat.960

Quero, F., Nogi, M., Lee, K. Y., Poel, G. V., Bismarck, A., Mantalaris, A., et al. (2011). Cross-linked bacterial cellulose networks using glyoxalization. ACS Appl. Mater. Interfaces 3, 490-499. doi: 10.1021/am101065p

Raafat, D., Bargen, K., Haas, A., and Sahl, H. G. (2008). Insights into the mode of action of chitosan as an antibacterial compound. Appl. Environ. Microbiol. 74, 3764-3773. doi: 10.1128/AEM.00453-08

Retegi, A., Gabilondo, N., Pena, C., Zuluaga, R., Castro, C., Ganan, P., et al. (2010). Bacterial cellulose films with controlled microstructure-mechanical property relationships. Cellulose 17, 661-669. doi: 10.1007/s10570-009-9389-7

Serafica, G., Mormino, R., Oster, G. A., Lentz, K. E., and Koehler, K. (2010). Microbial Cellulose Wound Dressing for Treating Chronic Wounds. US Patent, US 7390499. Washington, DC: U.S. Patent and Trademark Office.
Serafica, G. S., Mormino, R. M., and Bungay, H. B. (2002). Inclusion of solid particles in bacterial cellulose. Appl. Microbiol. Biotechnol. 58, 756-760. doi: 10.1007/s00253-002-0978-8

Singh, G., Joyce, E. M., Beddow, J., and Mason, T. J. (2012). Evaluation of antibacterial activity of $\mathrm{ZnO}$ nanoparticles coated sonochemically onto textile fabrics. J. Microbiol. Biotechnol. Food Sci. 2, 106-120. Available online at: http:// www.jmbfs.org/issue/august-september-2012-vol-2-no-1/jmbfs-0117-singh/? issue_id $=1307$ \&article_id $=6$

Tang, J., Bao, L., Li, X., Chen, L., and Hong, F. F. (2015). Potential of PVA-doped bacterial nano-cellulose tubular composites for artificial blood vessels. J. Mater. Chem. B 3, 8537-8547. doi: 10.1039/c5tb01144b

Ul-Islam, M., Shah, N., Ha, J., and Park, J. (2011). Effect of chitosan penetration on physico-chemical and mechanical properties of bacterial cellulose. Korean J. Chem. Eng. 28, 1736-1743. doi: 10.1007/s11814-011-0042-4

Verschuren, P. G., Cardona, T. D., Nout, M. J. R., De Gooijer, K. D., and Van den Heuvel, J. C. (2000). Location and limitation of cellulose production by Acetobacter xylinum established from oxygen profiles. J. Biosci. Bioeng. 89, 414-419. doi: 10.1016/s1389-1723(00)89089-1

Wei, B., Yang, G. A., and Hong, F. (2011). Preparation and evaluation of a kind of bacterial cellulose dry films with antibacterial properties. Carbohydr. Polym. 84, 533-538. doi: 10.1016/j.carbpol.2010.12.017

Wesarg, F., Schlott, F., Grabow, J., Kurland, H.-D., Heßler, N., Kralisch, D., et al. (2012). In situ synthesis of photocatalytically active hybrids consisting of bacterial nanocellulose and anatase nanoparticles. Langmuir 28, 13518-13525. doi: $10.1021 / 1 \mathrm{la} 302787 \mathrm{z}$

Wu, Y. B., Yu, S. H., Mi, F. L., Wu, C. W., Shyu, S. S., Peng, C. K., et al. (2004). Preparation and characterization on mechanical and antibacterial properties of chitosan/cellulose blends. Carbohydr. Polym. 57, 435-440. doi: 10.1016/j.carbpol.2004.05.013

Yan, Z., Chen, S., Wang, H., Wang, B., and Jiang, J. (2008). Biosynthesis of bacterial cellulose/multi-walled carbon nanotubes in agitated culture. Carbohydr. Polym. 74, 659-665. doi: 10.1016/j.carbpol.2008.04.028

Yang, X. Y., Huang, C., Guo, H. J., Xiong, L., Luo, J., Wang, B., et al. (2014). Beneficial effect of acetic acid on the xylose utilization and bacterial cellulose production by Gluconacetobacter xylinus. Indian J. Microbiol. 54, 268-273. doi: 10.1007/s12088-014-0450-3

Yang, Y. M., Zhao, Y. H., Liu, X. H., Ding, F., and Gu, X. S. (2007). The effect of different sterilization procedures on chitosan dried powder. J. Appl. Polym. Sci. 104, 1968-1972. doi: 10.1002/app.25906

Young, C. D., Wu, J. R., and Tsou, T. L. (1998). High-strength, ultra-thin and fiber-reinforced pHEMA artificial skin. Biomaterials 19, 1745-1752. doi: 10.1016/S0142-9612(98)00083-0

Zaborowska, M., Bodin, A., Bäckdahl, H., Popp, J., Goldstein, A., and Gatenholm, P. (2010). Microporous bacterial cellulose as a potential scaffold for bone regeneration. Acta Biomater. 6, 2540-2547. doi: 10.1016/j.actbio.2010. 01.004

Zhang, P., Tang, S. J., and Hong, F. (2013). Production of a bacterial cellulosecotton gauze blended composite film in a horizontal rotating bioreactor. Abstr. Pap. Am. Chem. Soc. 245. Available online at: http://acselb-529643017. us-west-2.elb.amazonaws.com/chem $/ 245 \mathrm{~nm} /$ program/view.php?obj_id=17264 9\&terms $=$

Conflict of Interest Statement: The authors declare that the research was conducted in the absence of any commercial or financial relationships that could be construed as a potential conflict of interest.

FFH is an author on patent applications on the construction of bioreactor and the in situ dynamic culture technology for biofabrication of chitosan/BNC composites.

Copyright $\odot 2016$ Zhang, Chen, Zhang and Hong. This is an open-access article distributed under the terms of the Creative Commons Attribution License (CC BY). The use, distribution or reproduction in other forums is permitted, provided the original author(s) or licensor are credited and that the original publication in this journal is cited, in accordance with accepted academic practice. No use, distribution or reproduction is permitted which does not comply with these terms. 